\title{
When Does Fusing Two Rings Not Yield a Larger Ring? The Curious Case of BOPHY
}

\section{Supporting Information}

Muhammed Buyuktemiz, ${ }^{a}$ Murat Kılıç, ${ }^{\mathrm{b}, \mathrm{c}}$ Yuanyuan Che, ${ }^{\mathrm{d}}$ Jianzhang Zhao, ${ }^{\mathrm{d}, \mathrm{e},{ }^{*}}$ and Yavuz Dede ${ }^{\mathrm{a},{ }^{*}}$

a. Department of Chemistry, Faculty of Science, Gazi University, Teknikokullar, 06500, Ankara, Turkey.

b. Van't Hoff Institute for Molecular Sciences, University of Amsterdam, Science Park 904, 1098 XH Amsterdam, The Netherlands

c. (present address) Institute of Chemical Sciences and Engineering, École Polytechnique Fédérale de Lausanne (EPFL), CH-1015 Lausanne, Switzerland

d. School of Chemistry, Xinjiang University, Urumqi 830046, Xinjiang, China.

e. State Key Laboratory of Fine Chemicals, School of Chemical Engineering, Dalian University of Technology, E-208 West Campus, 2 Ling Gong Rd., Dalian 116024, P. R. China. 


\section{Contents}

Pages

Scheme S1. Molecules discussed in this study

Figure S1. Optimized geometry of 1 with CAS(16,14)/6-31G(d) level of theory

Table S1. $S_{0} \rightarrow S_{1}$ Excitation Wavelength for 1 and $\mathbf{2}$ Using Various Levels of Theory

Measurement of Interplanar angle

Figure S2. BOPHY is dissected into two subunits, A and B

Figure S3. Interplanar angles of cubane. Hydrogens are omitted for clarity

Figure S4. Plots of planes passing through subunits A (left) and B (right)

Figure S5. Comparison of the Kohn-Sham energies and the interplanar angle

fluctuations of single BOPHY at $\mathrm{T}=300^{\circ} \mathrm{K}$ from DFT-MD calculations

Figure S6. Four BOPHY molecules were included in the DFT-MD simulation and periodicity mimicking the packing and $\pi$-stacking effects

Table S2. Molecular Orbitals and Natural Orbital Occupation Numbers (NOONs) for $\mathrm{S}_{0}$, $\mathrm{T}_{1}$ and $\mathrm{S}_{1}$ States of 1 at $\mathrm{CAS}(16,14) / 6-31 \mathrm{G}(\mathrm{d})$ Level of Theory

Table S3. Molecular Orbitals and Natural Orbital Occupation Numbers (NOONs) for So, $\mathrm{T}_{1}$ and $\mathrm{S}_{1}$ States of $\mathbf{2}$ at CAS $(10,8) / 6-31 \mathrm{G}(\mathrm{d})$ Level of Theory

Figure S7. Structural parameters of 1 for $S_{0}, T_{1}$, and $S_{1}$ geometries. Level of theory: $\operatorname{CAS}(16,14) / 6-31 G(d)$

Figure S8. TD-DFT spectrum of 1 using UB3LYP/cc-pVTZ level of theory

Figure S9. TD-DFT excitations of 1 using UB3LYP/cc-pVTZ level of theory

Figure S10. TD-DFT spectrum of TS1/1' using UB3LYP/cc-pVTZ level of theory

Figure S11. TD-DFT excitations of TS 1/1' using UB3LYP/cc-pVTZ level of theory Conical Intersection (CX)

Figure S12. (a) Optimized geometry of CX, (b) selected bond lengths of CX and SO equilibrium geometry for 1 using CAS/6-31G(d) level of theory

Table S4. Energy Differences for CX Geometry and the $S_{2}$ State

Figure S13. Relative energies of 1 for $S_{0}$ and $S_{1}$ states from FC geometry to $\mathrm{S} 1$ geometry (left) and from FC geometry to CX geometry (right)

Figure S14. Decay without any barrier along the TS1/1' to $S_{1}$ geometry on $S_{0}$ and $S_{1}$ surfaces

Figure S15. Natural orbital occupancies for $S_{0}$ and $S_{1}$ states at $S_{0}$ and $C X$ equilibrium geometries

Figure S16. TD-DFT spectrum of 2 using UB3LYP/cc-pVTZ level of theory with

Figure S17. TD-DFT excitations of $\mathbf{2}$ using UB3LYP/cc-pVTZ level of theory with

Figure S18. Optimized geometry of 1C with UB3LYP/6-31G(d) level of theory

Figure S19. Resonance structures of 1

Figure S20. TD-DFT spectrum of 3 using UB3LYP/cc-pVTZ level of theory

Figure S21. Structural modifications leading to avoid $\mathrm{N}=\mathrm{N}$ lone pair and red-shift of the absorption maximum

Figure S22. TD-DFT spectrum of 3a using UB3LYP/cc-pVTZ level of theory with

Table S5. Effect of Substitutions on BOPHY Core 
Figure S27. The molecular structure of compound 1-Me used in casting and polymer measurements

Figure S28. (a) UV-vis absorption of 1-Me casting film on quartz plates. (b) Comparison of the UV-Vis absorption of the thick film and the thin film

Figure S29. UV-vis absorption of (a) 1-Me doped in the polymer film and (b) blank polymer film at $20^{\circ} \mathrm{C}$

Polymer Film Preparation 
Scheme S1. Molecules discussed in this study

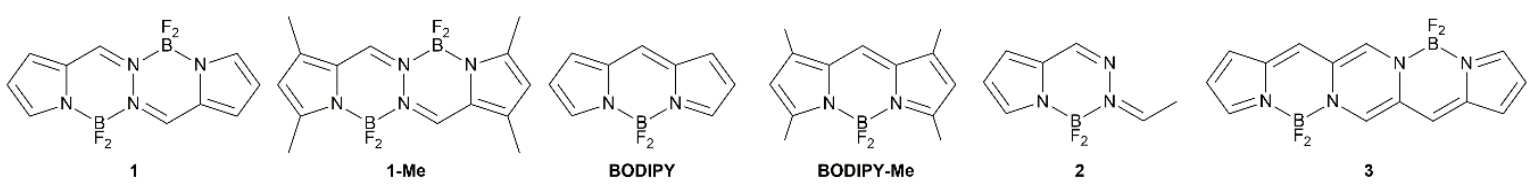

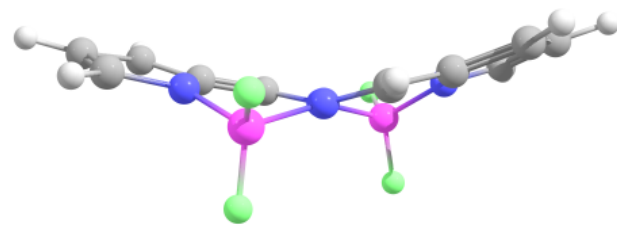

$\mathbf{H}$
$\mathbf{B}$
$\mathbf{N}$
$\mathbf{C}$
$\mathbf{F}$
Front

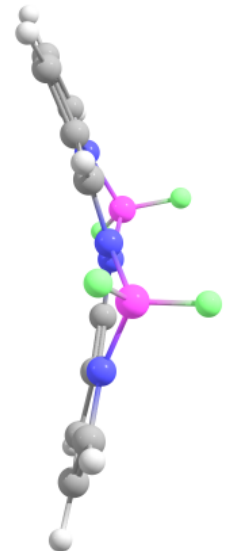

Side

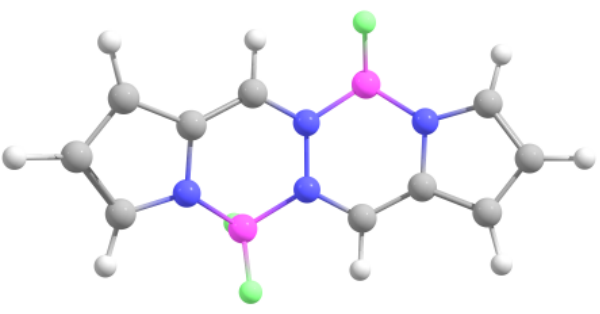

Top

Figure S1. Optimized geometry of 1 with $\operatorname{CAS}(16,14) / 6-31 \mathrm{G}(\mathrm{d})$ level of theory. Various functional/basis set combinations are used in DFT and multiple active spaces with different electron/orbital configurations are considered for CASSCF runs. See main text for computational details. 
Table S1. $S_{0} \rightarrow S_{1}$ Excitation Wavelength for 1 and 2 Using Various Levels of Theory

\begin{tabular}{|l|r|r|}
\hline & $\mathbf{1}$ & $\mathbf{2}$ \\
\hline Level of Theory & $\begin{array}{l}\mathbf{S}_{\mathbf{1}} \\
\mathbf{( n m})\end{array}$ & $\begin{array}{l}\mathbf{S}_{\mathbf{1}} \\
\mathbf{( n m})\end{array}$ \\
\hline a UMP2/6-31G(d) & 315 & 276 \\
\hline aUMP2/cc-pVTZ & 321 & 314 \\
\hline a $\omega \omega B 97 X D / 6-31 G(d)$ & 354 & 280 \\
\hline a $\omega \omega B 97 X D / c c-p V T Z$ & 358 & 313 \\
\hline a UM06-2X/6-31G(d) & 359 & 319 \\
\hline a UM06-2X/cc-pVTZ & 361 & 319 \\
\hline a UB3LYP/6-31G(d) & 391 & 356 \\
\hline aUB3LYP/cc-pVTZ & 386 & 355 \\
\hline CAS/6-31G(d) & 322 & 277 \\
\hline CAS-PT2/6-31G(d) & 332 & 329 \\
\hline EOM-CCSD/6-31G(d) & 353 & 306 \\
\hline EOM-CCSD/cc-pVTZ & 356 & 310 \\
\hline Exp. & $424^{b}$ & $\mathrm{n} / \mathrm{a}$ \\
\hline
\end{tabular}

${ }^{a}$ Excitation is studied with time dependent variant of the same method. ${ }^{\mathrm{b}} \mathrm{A}$ doublet peak is measured in the experimental spectrum. 


\section{Measurement of Interplanar angle}

A simple least square solution is applied to the cartesian coordinates of $\mathbf{1}$ in order to show the degree of puckering in a qualitative manner. On the outset, $\mathbf{1}$ is dissected into two separate units. Dissection plane was chosen to include $\mathrm{N}-\mathrm{N}$ bond at the center of the molecule. Corresponding two subunits are labeled as $A$ and $B$ (Figure $\mathrm{S} 2$ ). Considering the large deviation of the $\mathrm{BF}_{2}$ moieties in the trans conformation, $\mathrm{BF}_{2}$ 's are disregarded in the computation of the angle between $\mathrm{A}$ and $\mathrm{B}$ for both units. Interplanar angle is then calculated using the equations given below.

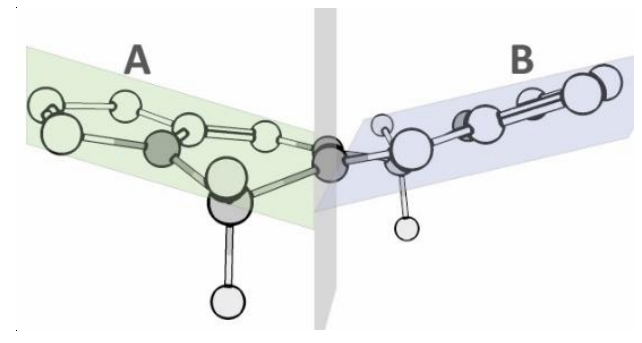

Figure S2. BOPHY is dissected into two subunits, $A$ and $B$. Planes passing through subunits $A$ and $B$ are fitted using respective cartesian coordinates according to equation $1-4$ (vide infra). Hydrogen atoms are omitted for clarity.

Equation for a plane is;

$$
a x+b y+c=z
$$

In the matrix form, equation (1) can be transformed as;

$$
\begin{gathered}
{\left[\begin{array}{ccc}
x_{1} & y_{1} & 1 \\
\vdots & \ddots & \vdots \\
x_{n} & y_{n} & 1
\end{array}\right]\left[\begin{array}{l}
a \\
b \\
c
\end{array}\right]=\left[\begin{array}{c}
z_{1} \\
\vdots \\
z_{n}
\end{array}\right]} \\
A x=B
\end{gathered}
$$

Solving for $x$, which is the coefficients, gives the desired equation for a plane. However, since the number of points in the molecule 1 is more than 3 , the system is over-determined. By using leftpseudo inverse in this case, the number of points are fitted into a plane;

$$
\left[\begin{array}{l}
a \\
b \\
c
\end{array}\right]=\left(A^{T} A\right)^{-1} A^{T} B
$$

The validity of the method described above was tested with cubic-shaped Cubane molecule (Figure S3). Angle between selected planes $(\alpha)$ were found to be 45.0 degrees with a fitting error of $10^{-15}$.

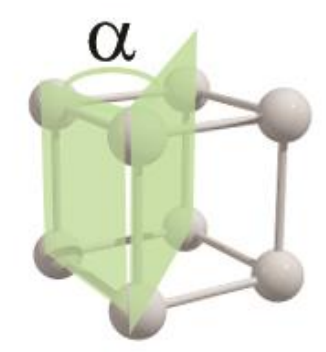

Figure S3. Interplanar angles of cubane. Hydrogens are omitted for clarity. 
Using CASSCF optimized geometries, interplanar angle of subunits A and B are calculated.

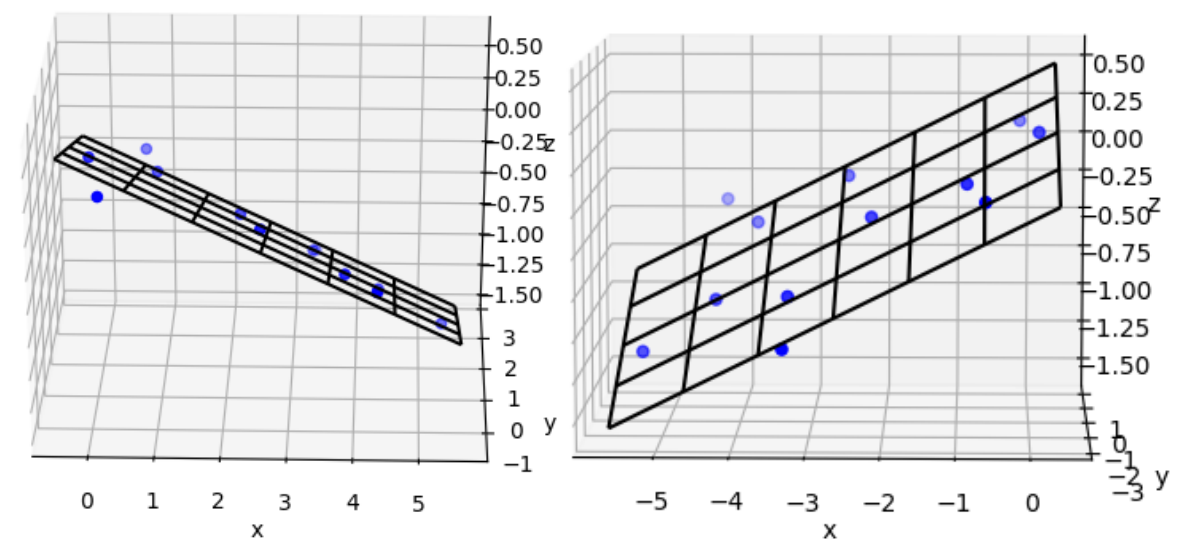

Figure S4. Plots of planes passing through subunits A (left) and B (right).

Given the equations for the planes $A$ and B, angle between them is calculated using Eq. (5);

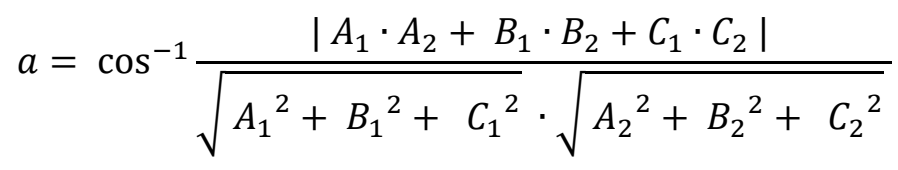




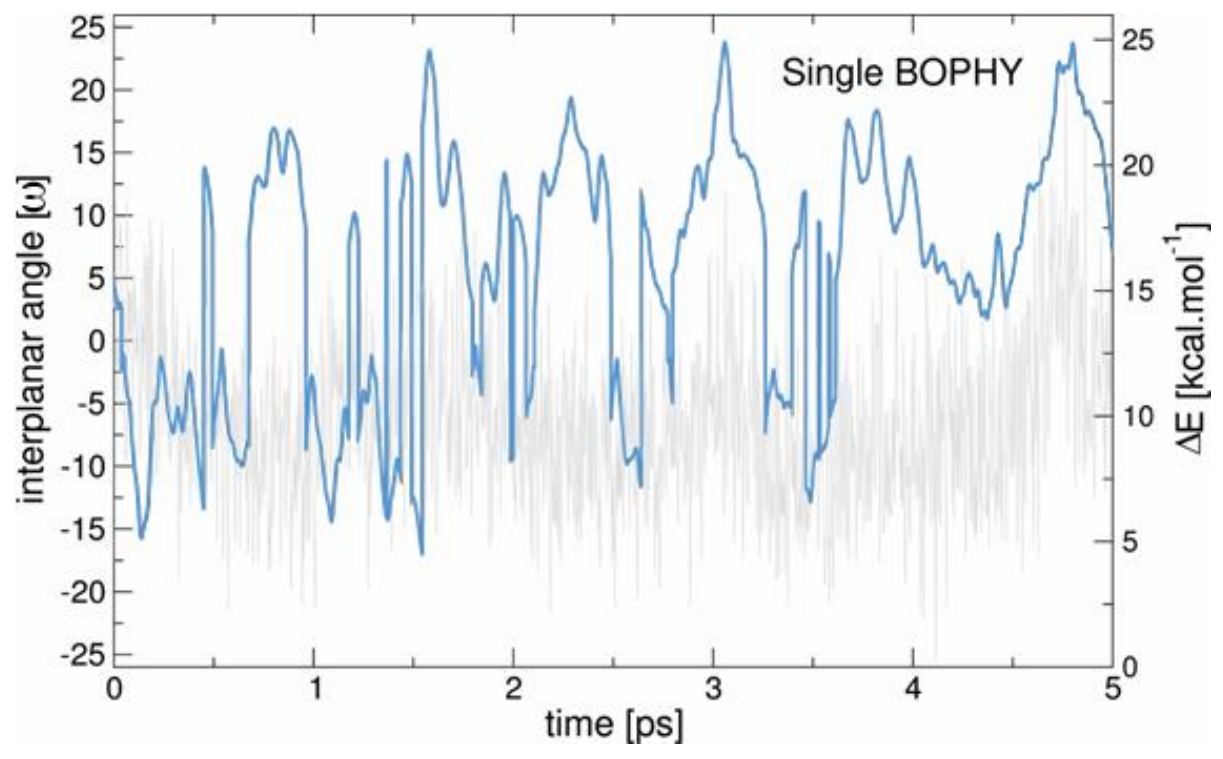

Figure S5. Comparison of the Kohn-Sham energies (gray line) and the interplanar angle fluctuations (blue line) of single BOPHY at T=300 K from DFT-MD calculations.

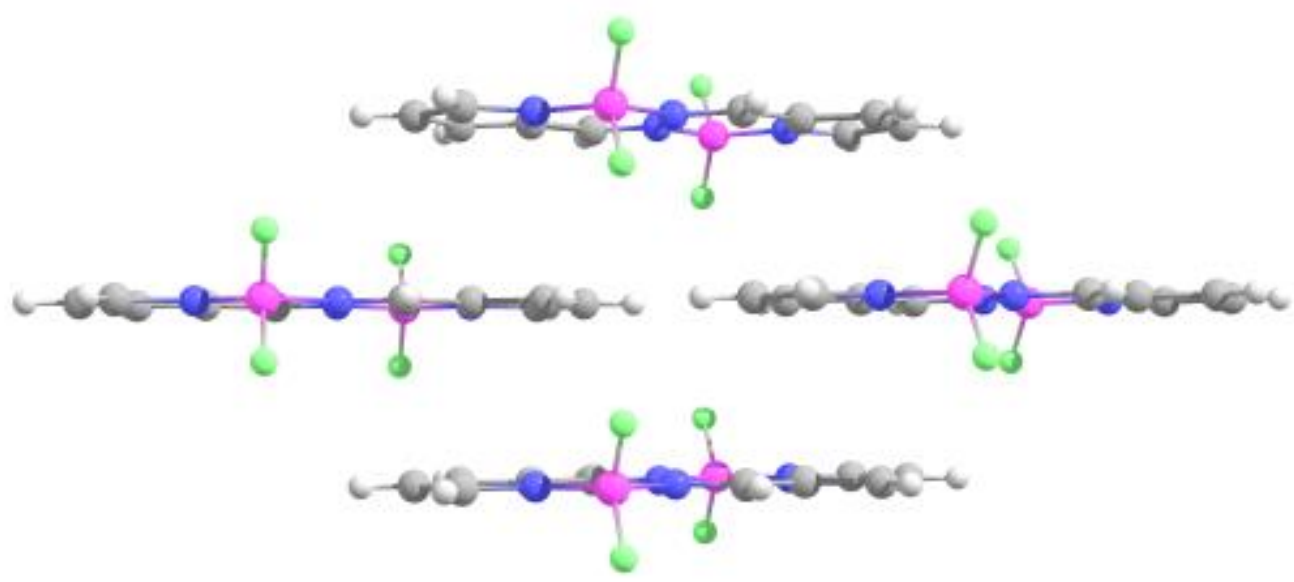

Figure S6. Four BOPHY molecules were included in the DFT-MD simulation and periodicity mimicking the packing and $\pi$-stacking effects. 
Table S2. Molecular Orbitals and Natural Orbital Occupation Numbers (NOONs) for $\mathrm{S}_{0}, \mathrm{~T}_{1}$ and $\mathrm{S}_{1}$ States of 1 at CAS(16,14)/6-31G(d) Level of Theory

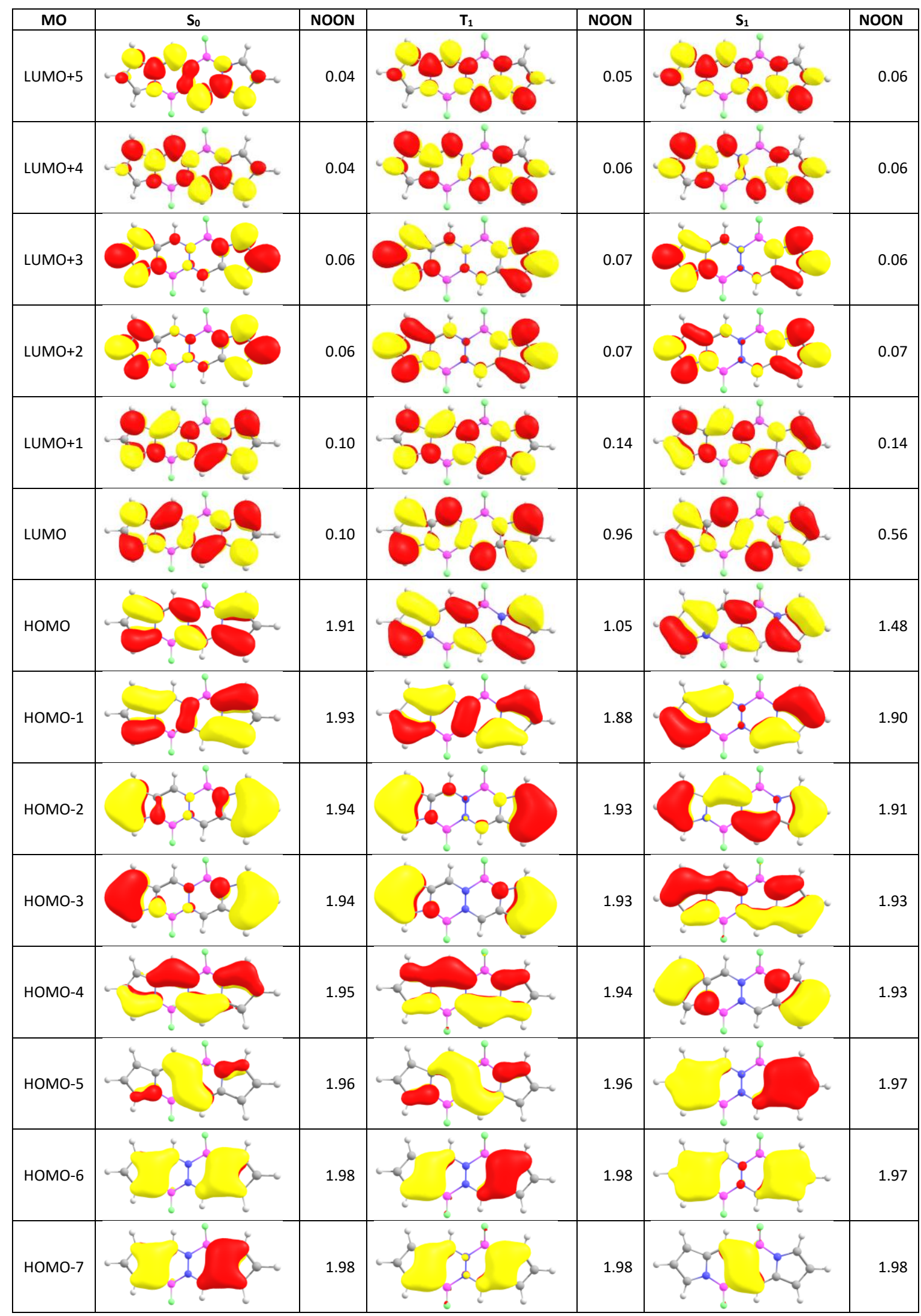


Table S3. Molecular Orbitals and Natural Orbital Occupation Numbers (NOONs) for $\mathrm{S}_{0}, \mathrm{~T}_{1}$ and $\mathrm{S}_{1}$ States of 2 at CAS $(10,8) / 6-31 G(d)$ Level of Theory

\begin{tabular}{|c|c|c|c|c|c|c|}
\hline MO & $S_{0}$ & NOON & $\mathrm{T}_{1}$ & NOON & $S_{1}$ & NOON \\
\hline LUMO+2 & & 0.05 & & 0.06 & & 0.06 \\
\hline LUMO+1 & & 0.08 & & 0.09 & & 0.12 \\
\hline LUMO & & 0.09 & & 0.97 & & 0.64 \\
\hline Hомо & & 1.90 & & 1.03 & & 1.37 \\
\hline HOMO-1 & & 1.94 & & 1.91 & & 1.89 \\
\hline HOMO-2 & & 1.96 & & 1.95 & & 1.95 \\
\hline HOMO-3 & & 1.99 & & 1.99 & & 1.99 \\
\hline HOMO-4 & & 1.99 & & 1.99 & & 1.99 \\
\hline
\end{tabular}

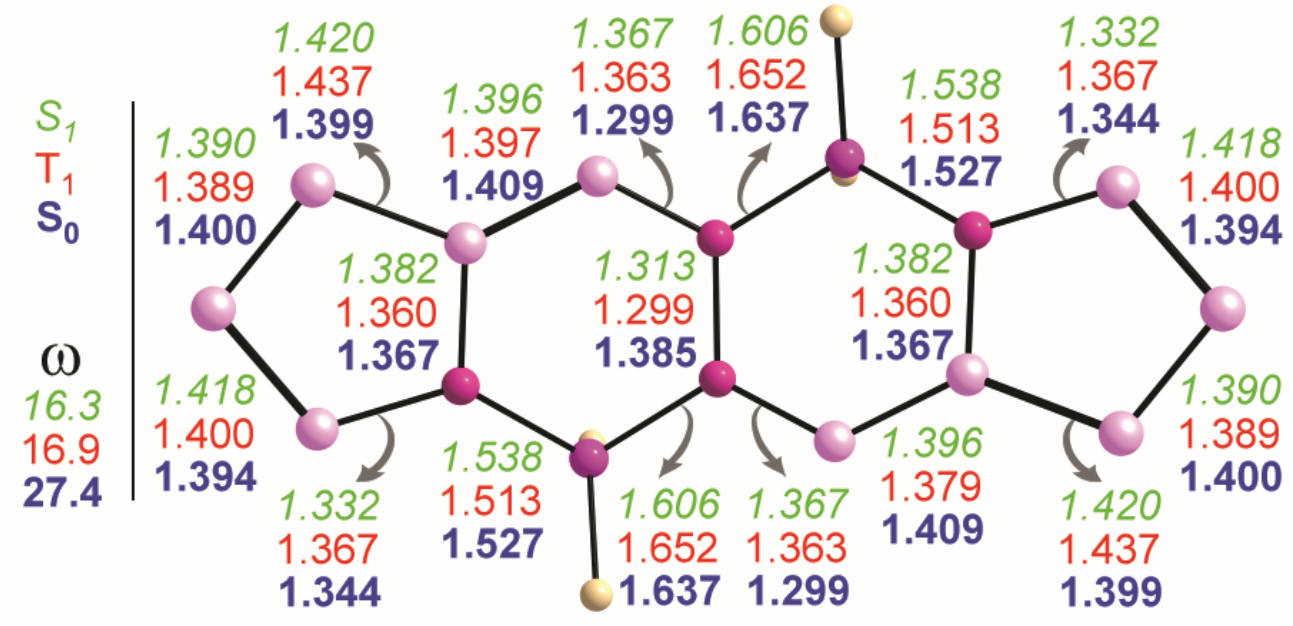

Figure S7. Structural parameters of 1 for $S_{0}, T_{1}$, and $S_{1}$ geometries. Level of theory: $C A S(16,14) / 6$ $31 \mathrm{G}(\mathrm{d})$. 


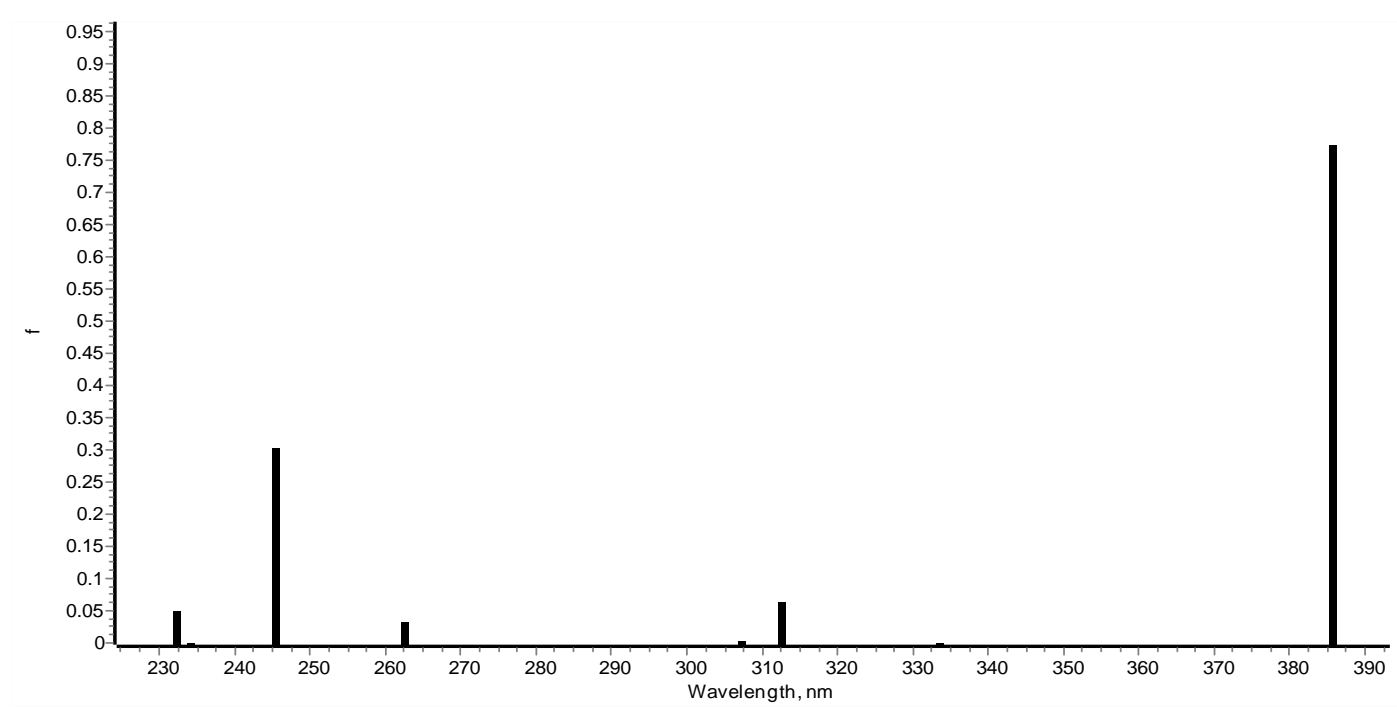

Figure S8. TD-DFT spectrum of 1 using UB3LYP/cc-pVTZ level of theory with dichloromethane as solvent.
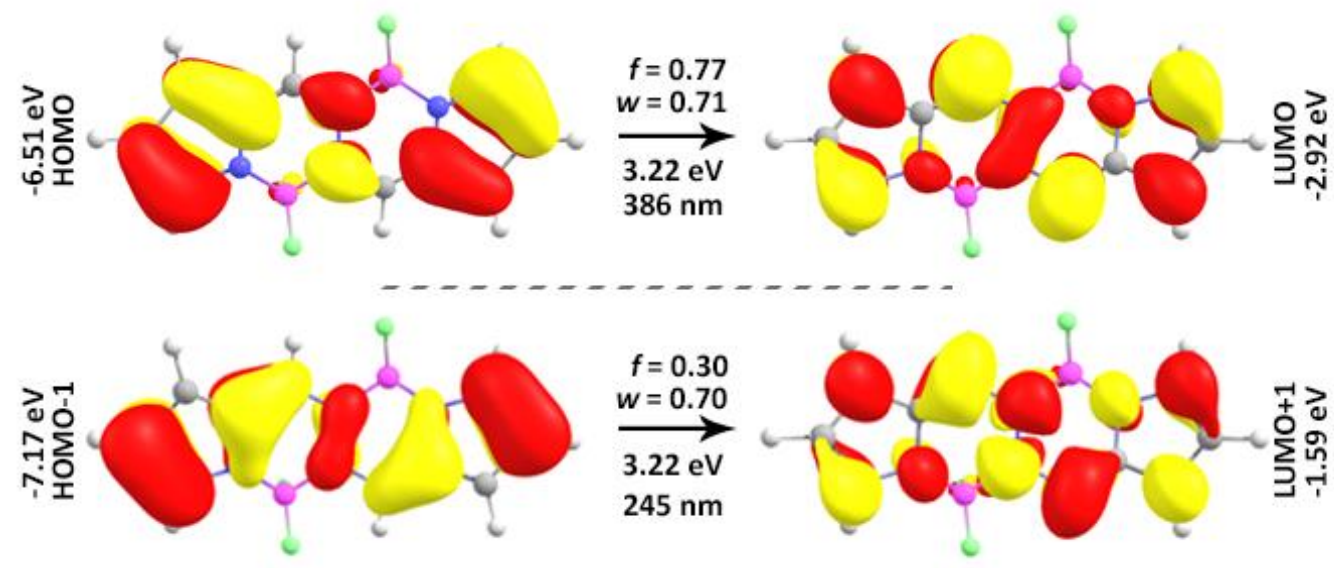

Figure S9. TD-DFT excitations of 1 using UB3LYP/cc-pVTZ level of theory with dichloromethane as solvent. Oscillator strengths smaller than 0.06 are not considered. $f$ : oscillator strength, $w$ : weight.

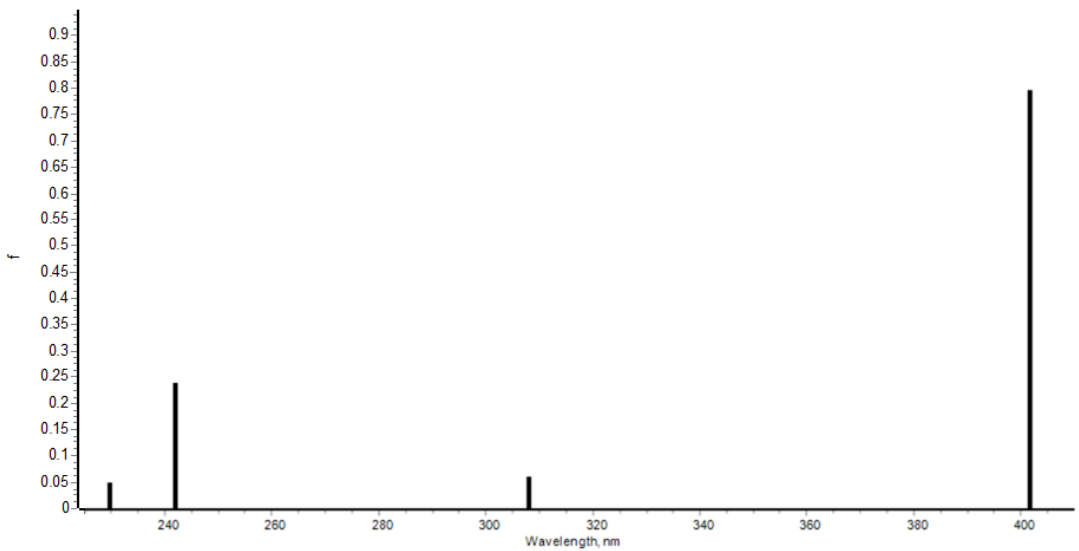

Figure S10. TD-DFT spectrum of TS1/1' using UB3LYP/cc-pVTZ level of theory with dichloromethane as solvent. 


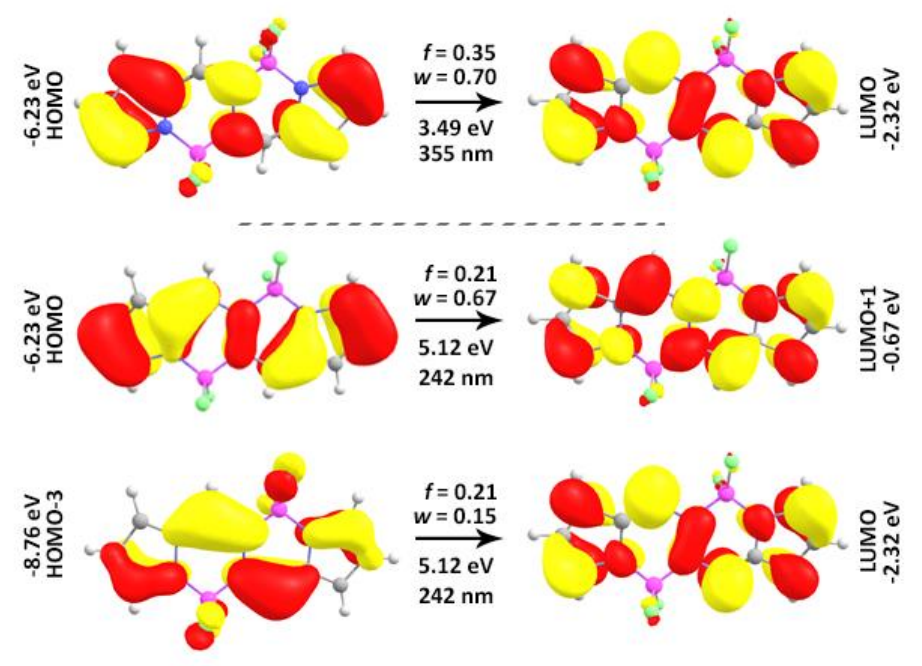

Figure S11. TD-DFT excitations of TS 1/1' using UB3LYP/cc-pVTZ level of theory with dichloromethane as solvent. Oscillator strengths smaller than 0.09 are not considered. $f$ : oscillator strength, w: weight.

\section{Conical Intersection (CX)}

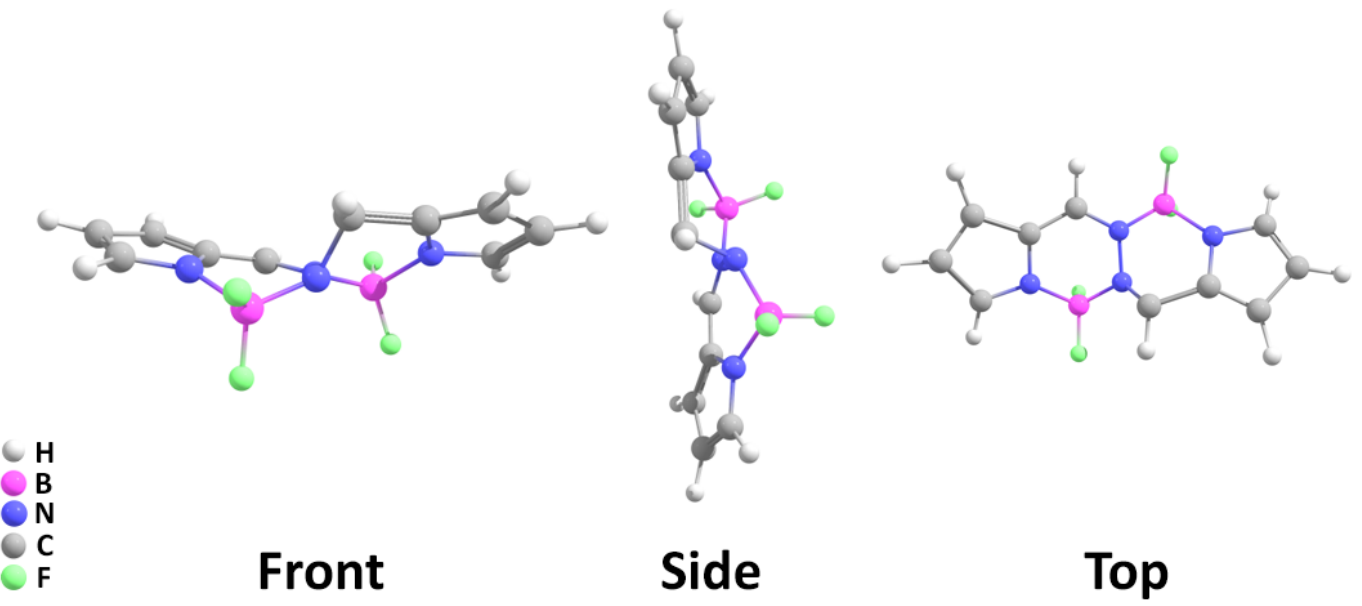

(a)
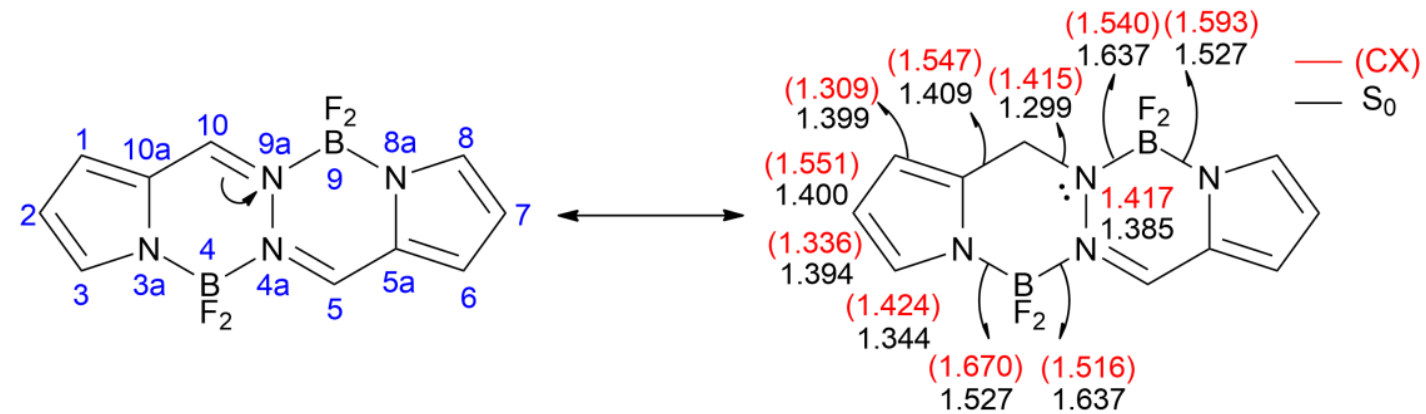

(b)

Figure S12. (a) Optimized geometry of $C X$, (b) selected bond lengths of $C X$ and $S_{0}$ equilibrium geometry for 1 using CAS/6-31G(d) level of theory. 
Table S4. Energy Differences for CX Geometry and the $S_{2}$ State

\begin{tabular}{|l|l|l|l|l|l|}
\hline & $\mathrm{S}_{1} @ C X-\mathrm{S}_{1} @ \mathrm{~S}_{0}$ & $\mathrm{~S}_{1} @ C X-\mathrm{S}_{0} @ \mathrm{~S}_{0}$ & $\mathrm{~S}_{1} @ C X-\mathrm{S}_{0} @ C X$ & $\mathrm{~S}_{2} @ \mathrm{~S}_{0}-\mathrm{S}_{0}$ & $\mathrm{~S}_{2} @ \mathrm{~S}_{0}-\mathrm{S}_{1} @ \mathrm{~S}_{0}$ \\
\hline $\mathrm{CAS}(16,14) / 6-31 \mathrm{G}(\mathrm{d})$ & $0.55 \mathrm{eV}$ & $4.40 \mathrm{eV}$ & $0.05 \mathrm{eV}$ & $4.49 \mathrm{eV}$ & $0.84 \mathrm{eV}$ \\
\hline CAS-PT2/6-31G(d) & $0.87 \mathrm{eV}$ & $4.61 \mathrm{eV}$ & $0.25 \mathrm{eV}$ & - & - \\
\hline
\end{tabular}

$\mathrm{S}_{1} @ C X: \mathrm{S}_{1}$ energy at CX geometry

So@CX: So energy at CX geometry

$\mathrm{S}_{1} @ \mathrm{~S}_{0}: \mathrm{S}_{1}$ energy at So geometry

So@S: So energy at So geometry
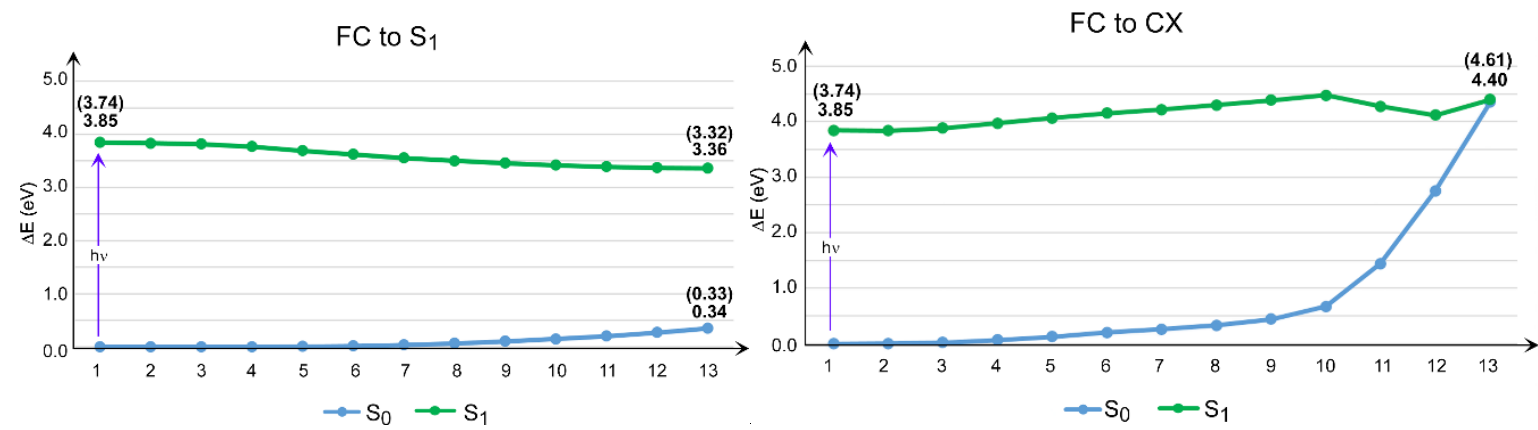

Figure S13. Relative energies of 1 for $S_{0}$ and $S_{1}$ states from FC geometry to $S_{1}$ geometry (left) and from FC geometry to CX geometry (right) calculated at CAS/6-31G(d) level of theory. CAS-PT2 energies are given in paranthesis.

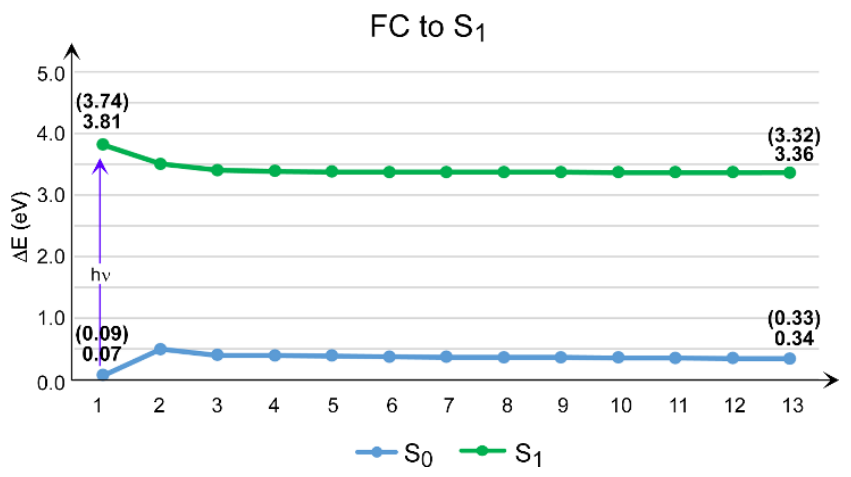

Figure S14. Decay without any barrier along the $T S 1 / 1^{\prime}$ to $S_{1}$ geometry on $S_{0}$ and $S_{1}$ surfaces calculated at CAS/6-31G(d) level of theory. CAS-PT2 energies are given in paranthesis. 


\begin{tabular}{|c|c|c|c|c|c|c|}
\hline & $s_{0}$ & $\mathrm{~s}_{1} @ \mathrm{~s}_{0}$ & & $s_{0} @ c x$ & $\mathrm{~s}_{1} @ \mathrm{cx}$ & \\
\hline$n c$ & 0.90 & 0.840 .170 .12 & $\begin{array}{c}\text { NOON } \\
\text { (SA) }\end{array}$ & $\begin{array}{l}0.780 .320 .240 .120 .120 .10\end{array}$ & 0.820 .320 .120 .110 .11 & $\begin{array}{c}\mathrm{NOON} \\
(\mathrm{SA})\end{array}$ \\
\hline nO+5 & - & --- & 0.05 & ----- & ----- & 0.04 \\
\hline $\mathrm{MO}+4$ & - & --- & 0.06 & ------ & ---- & 0.06 \\
\hline LUMO+3 & - & --- & 0.07 & ----- & ----- & 0.08 \\
\hline$-U M O+2$ & - & --- & 0.07 & ----- & --+-- & 0.09 \\
\hline LUMO+1 & - & $-t+$ & 0.13 & ----- & $---4-$ & 0.09 \\
\hline LUMO & - & $+-t$ & 0.53 & $-+4+4+4$ & $+4+t-$ & 0.73 \\
\hline номо & 斗 & $+4+$ & 1.50 & 世 4 + 4 4 4 & 斗 t+ tw t4 & 1.43 \\
\hline HOMO-1 & 斗 & $4+4$ & 1.90 & $4+4-4-1$ & $t+t+4$ & 1.74 \\
\hline номо-2 & 年 & 开 $4+$ & 1.92 & $4+-4+4$ & $4+4+4$ & 1.92 \\
\hline номо-3 & 4 & 4t 4 4 & 1.93 & स 4 स 4 स & t 4 t $4-$ - t & 1.94 \\
\hline номо-4 & 4 & 4t 4 t & 1.93 & $4+4+4+4$ & $4+4+4$ & 1.95 \\
\hline HOMO-5 & 4 & 4 4 年 & 1.97 & 出 4 4 4 4 4 & 出 $+4+4$ & 1.96 \\
\hline HOMO-6 & 4 & 4t 4 t & 1.97 & $4+4+4+4+4$ & 4t 4 t 4 t & 1.98 \\
\hline номо-7 & 4 & $44+4$ & 1.97 & 4t 4 t 4 t 4 t & 4t 4 +4 4 t & 1.99 \\
\hline
\end{tabular}

Figure S15. Natural orbital occupancies for $S_{0}$ and $S_{1}$ states at $S_{0}$ and $C X$ equilibrium geometries.

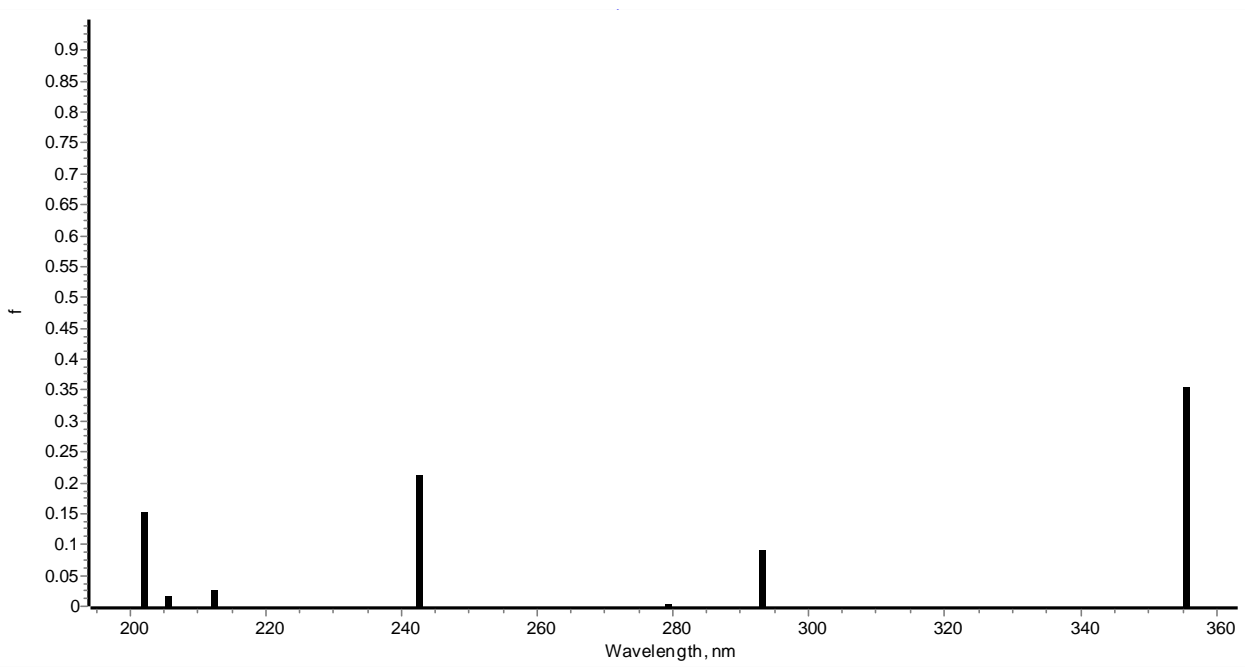

Figure S16. TD-DFT spectrum of 2 using UB3LYP/cc-pVTZ level of theory with dichloromethane as solvent. 

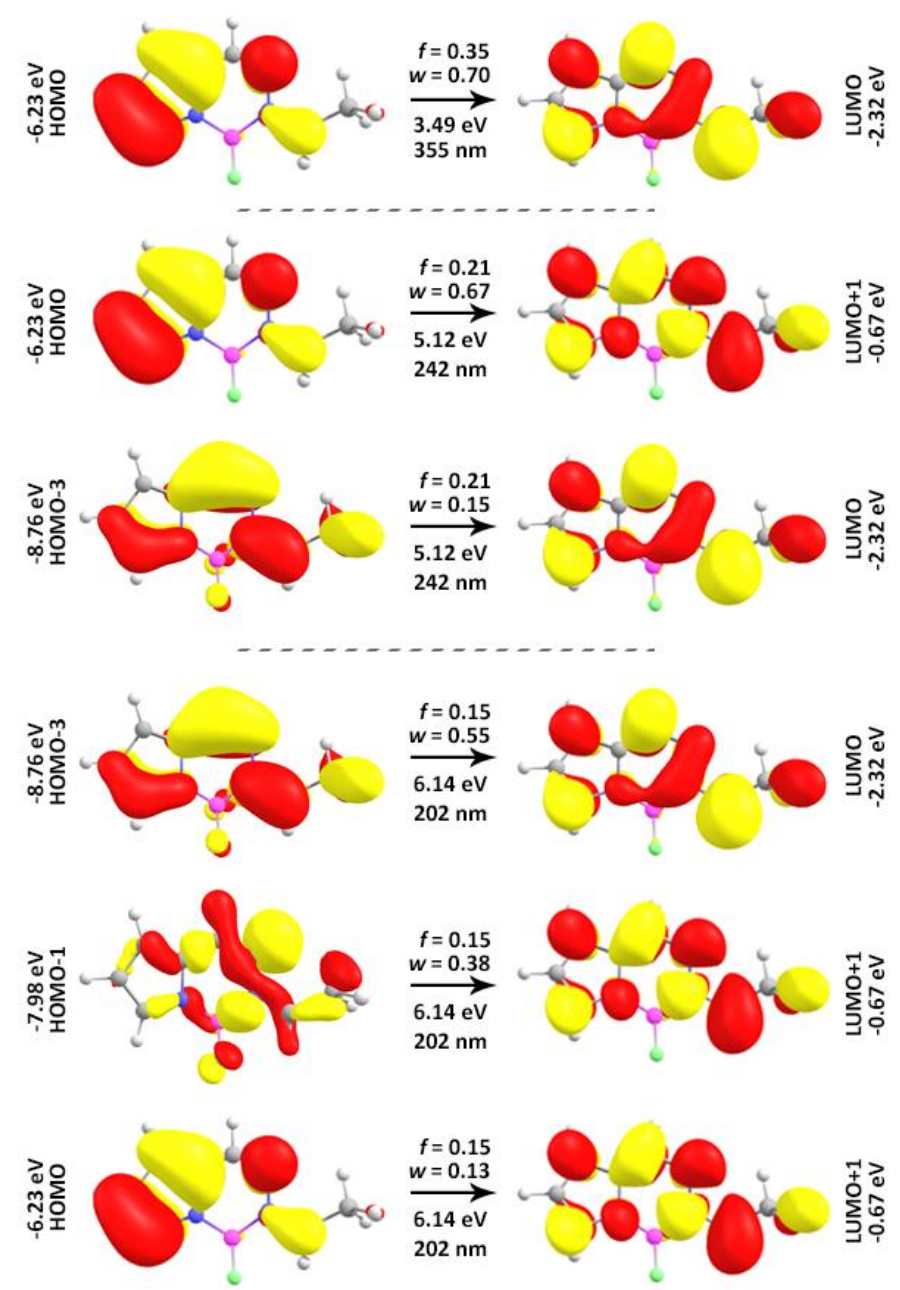

Figure S17. TD-DFT excitations of $\mathbf{2}$ using UB3LYP/cc-pVTZ level of theory with dichloromethane as solvent. Oscillator strengths smaller than 0.09 are not considered. $f$ : oscillator strength, w: weight. 


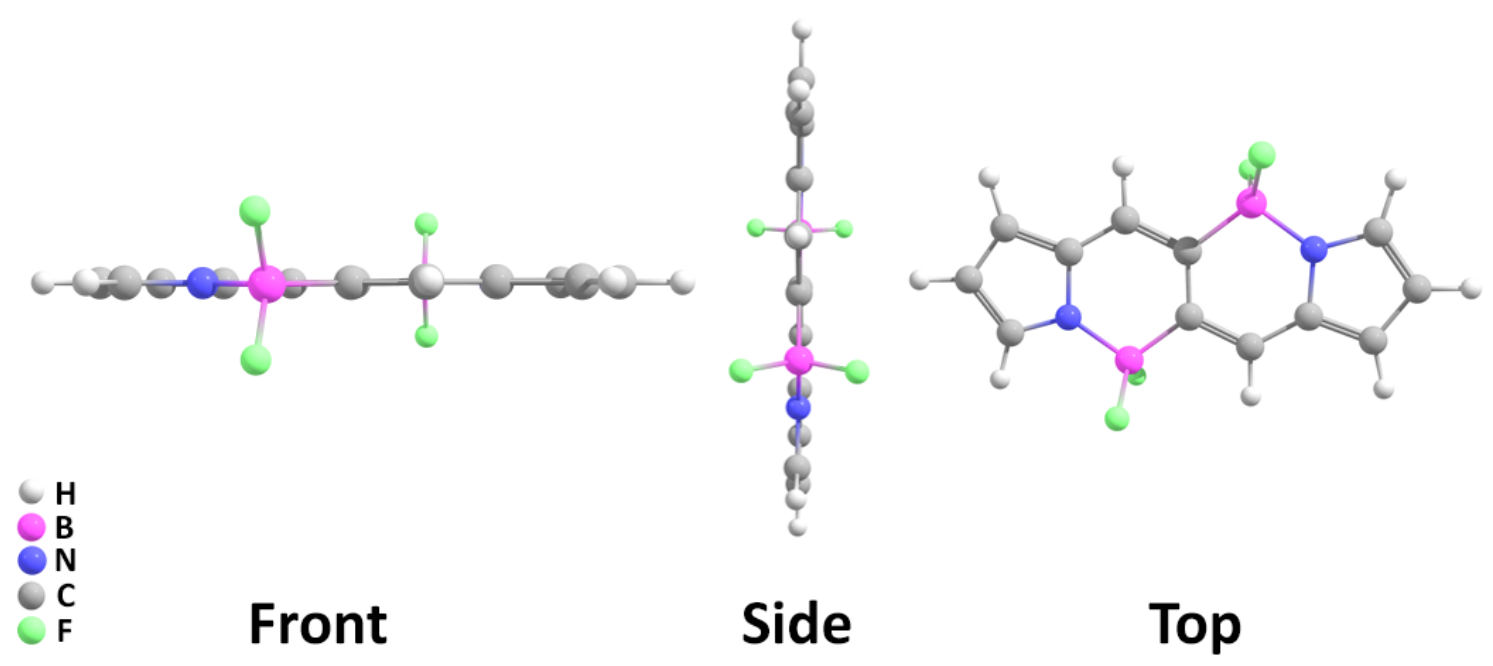

Figure S18. Optimized geometry of 1C with UB3LYP/6-31G(d) level of theory.

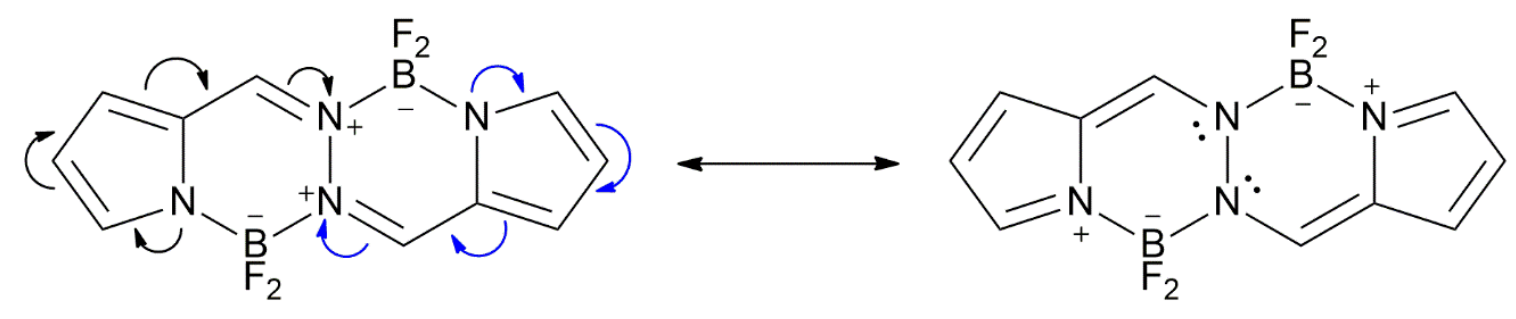

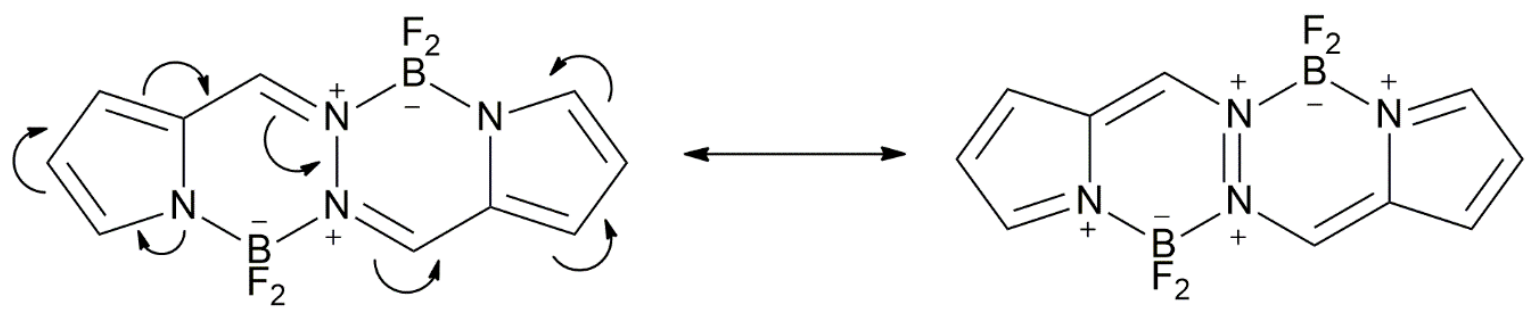

Figure S19. Resonance structures of 1.

Other resonance structures for $\mathbf{1}$ are also possible. Note that for the $\mathrm{N}-\mathrm{N}$-bridge either a lone pair repulsion or a formal positive charge accumulation is contributing to the resonance hybrid. 


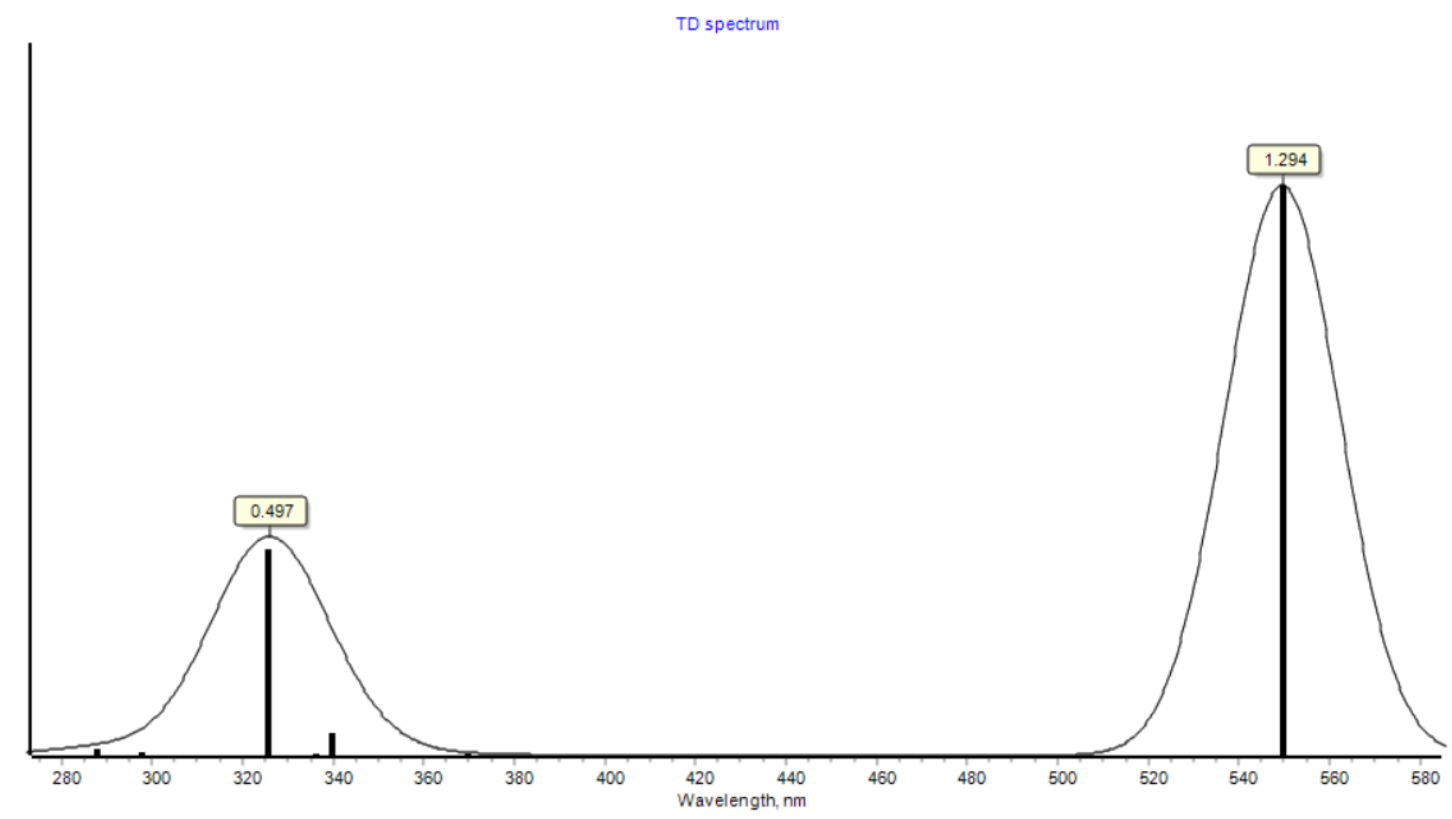

Figure S20. TD-DFT spectrum of $\mathbf{3}$ using UB3LYP/cc-pVTZ level of theory with dichloromethane as solvent.

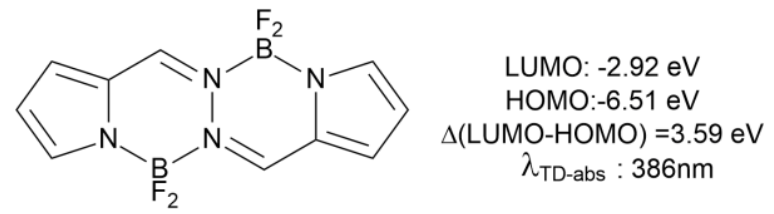
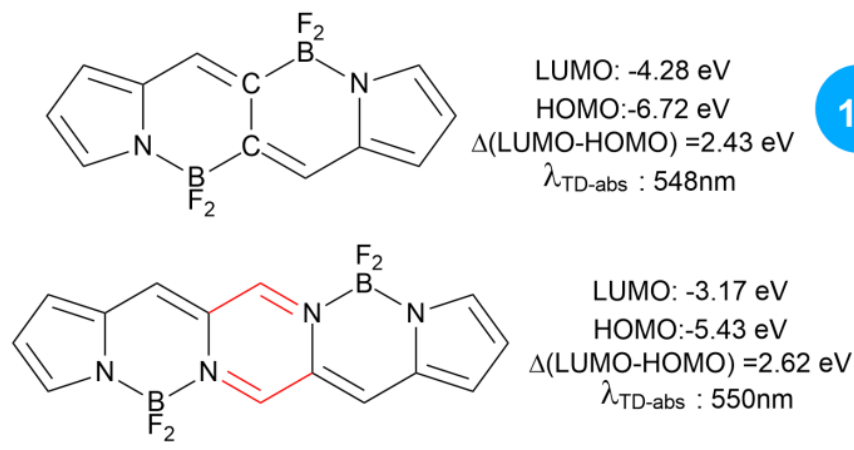

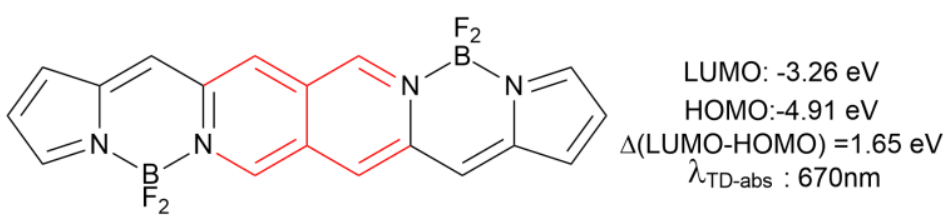

Figure S21. Structural modifications leading to avoid $\mathrm{N}=\mathrm{N}$ lone pair and red-shift of the absorption maximum. Frontier orbital energies are calculated using UB3LYP/cc-pVTZ level of theory with dichloromethane as solvent. 


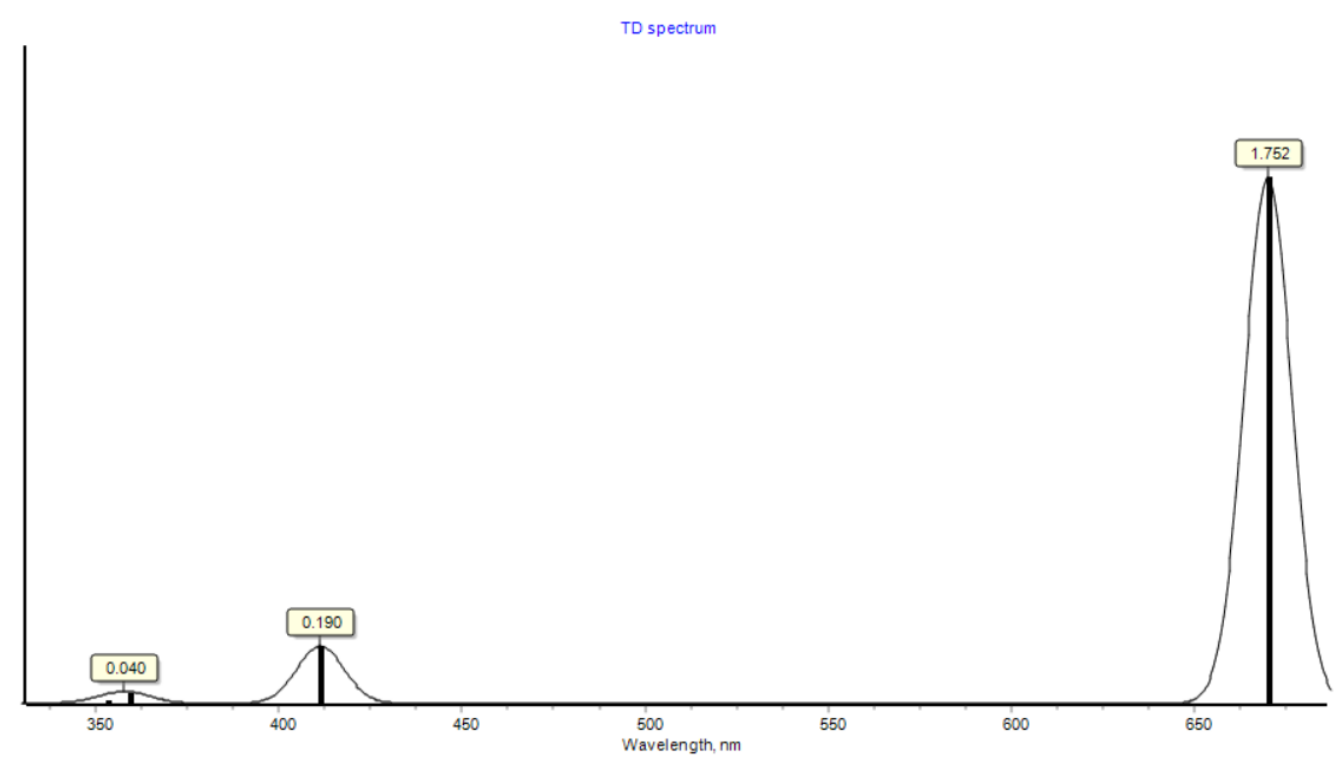

Figure S22. TD-DFT spectrum of 3a using UB3LYP/cc-pVTZ level of theory with dichloromethane as solvent.

Table S5. Effect of Substitutions on BOPHY Core

\begin{tabular}{|c|c|c|c|c|c|c|c|c|}
\hline & & $R_{1}$ & & $R_{3}$ & $\begin{array}{l}\mathrm{F}_{2} \\
\mathrm{~B}^{2}\end{array}$ & & $-R_{2}$ & \\
\hline $\begin{array}{l}\text { Interplanar } \\
\text { Angle } \\
\left({ }^{\circ}\right)\end{array}$ & $\mathbf{R}_{1}, \mathbf{R}_{\mathbf{2}}$ & $\mathbf{R}_{\mathbf{3}}, \mathbf{R}_{\mathbf{4}}$ & $\begin{array}{c}\Delta \mathrm{E}_{\left(\mathrm{TS} 1 \mid 1^{\prime}-1\right)} \\
(\mathrm{kcal} / \mathrm{mol})\end{array}$ & $\begin{array}{l}V_{\text {imag }} \\
\left(\mathrm{cm}^{-1}\right)\end{array}$ & $\begin{array}{c}\lambda_{\max } \\
\text { (Puckered) }^{\mathrm{a}} \\
(\mathrm{nm})\end{array}$ & $\begin{array}{c}\lambda_{\max } \\
\text { (Planar) } \\
(\mathrm{nm})\end{array}$ & $\begin{array}{c}\Delta \mathrm{E}_{\text {(LUMо-номо) }} \\
\text { (Puckered) } \\
\text { (eV) }\end{array}$ & $\begin{array}{c}\Delta \mathrm{E}_{\text {(LUMо-номо) }} \\
\text { (Planar) } \\
(\mathrm{eV})\end{array}$ \\
\hline 28.4 & $-\mathrm{H}$ & $-\mathrm{H}$ & 2.0 & 34 & 397 & 405 & 3.47 & 3.39 \\
\hline 31.2 & $-\mathrm{H}$ & $-\mathrm{CN}$ & 1.0 & 8 & 477 & 492 & 2.85 & 2.87 \\
\hline 36.2 & $-\mathrm{H}$ & $-\mathrm{NO}_{2}$ & 3.6 & 45 & 385 & 402 & 3.26 & 3.25 \\
\hline 31.7 & $-\mathrm{H}$ & $-\mathrm{COCH}_{3}$ & 3.9 & 50 & 409 & 412 & 3.23 & 3.36 \\
\hline 48.8 & $-\mathrm{H}$ & $-\mathrm{NH}_{2}$ & 4.7 & 87 & 318 & 332 & 4.48 & 4.31 \\
\hline 28.3 & $-\mathrm{CN}$ & $-\mathrm{H}$ & 2.3 & 29 & 396 & 405 & 3.56 & 3.46 \\
\hline 29.0 & $-\mathrm{NO}_{2}$ & $-\mathrm{H}$ & 2.3 & 29 & 398 & 406 & 3.52 & 3.44 \\
\hline 28.2 & $-\mathrm{COCH}_{3}$ & $-\mathrm{H}$ & 2.2 & 27 & 400 & 408 & 3.51 & 3.42 \\
\hline 26.2 & $-\mathrm{NH}_{2}$ & $-\mathrm{H}$ & 1.8 & 31 & $486 / 362$ & $491 / 367$ & 2.92 & 2.87 \\
\hline 27.3 & $\begin{array}{l}\mathrm{R}_{1}:-\mathrm{NH}_{2} \\
\mathrm{R}_{2}:-\mathrm{NO}_{2}\end{array}$ & $-\mathrm{H}$ & 2.2 & 31 & $533 / 387$ & $529 / 387$ & 2.76 & 2.75 \\
\hline
\end{tabular}

${ }^{a}$ UB3LYP/6-31G(d) level of theory is used for optimization/frequency calculations and UB3LYP/cC$\mathrm{pVTZ//UB3LYP/6-31G(d)} \mathrm{is} \mathrm{used} \mathrm{for} \mathrm{TD-DFT} \mathrm{and} \mathrm{single} \mathrm{point} \mathrm{energy} \mathrm{runs.}$ 


\section{Half-BOPHY}

Recently, Hao and co-workers synthesized polybrominated BOPHY dyes. ${ }^{1}$ Among the reported dyes, 4 and 5 (Figure S12) are particularly interesting in terms of their structural, electronic and optical properties.

Quantum fluorescence yield of $\mathbf{4}$ is measured as 0.69 . Synthesis and stability of $\mathbf{5}$ is also reported and it has a fluorescence yield of 0.24 . Excitation characters of both compounds are studied with TD-DFT using UB3LYP/6-31G(d) level of theory (Fig. S15-S16). The relationship between oscillator strengths and the quantum yields for compounds $\mathbf{4}$ and $\mathbf{5}$ supports the discussion in the main text; the optical properties of $\mathbf{4}$ is reminiscent of its modified subunit $\mathbf{5}$ and $\mathbf{4}$ has a larger oscillator strength and quantum yield.

We have also studied compound 6, the half of 4 with TD-DFT. Removal of $\mathrm{NH}\left(\mathrm{CH}_{2}\right)_{3} \mathrm{CH}_{3}$ moiety further decreases the oscillator strengths.<smiles></smiles>

$\Phi_{f}: 0.69$

$(465 \mathrm{~nm}, 488 \mathrm{~nm})$<smiles></smiles>

$\Phi_{f}: 0.24$

$(522 \mathrm{~nm}, 552 \mathrm{~nm})$<smiles>C=[N+]1N=Cc2c(Br)c(Br)c(Br)n2P1</smiles>

6

Figure S23. Polybrominated BOPHY and brominated half BOPHY. Fluorescence yields and absorption maxima are taken from Ref 23.

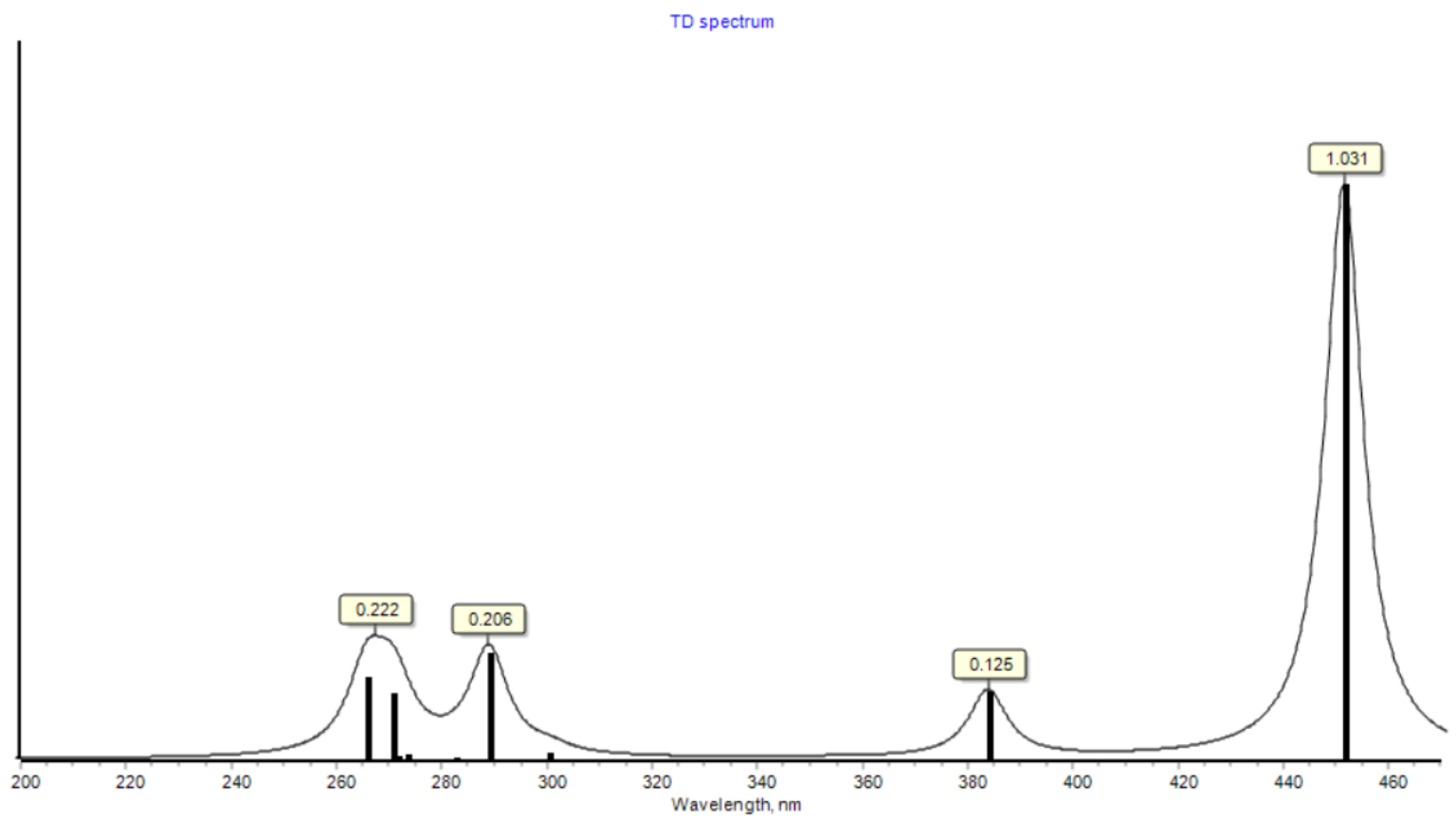

Figure S24. TD-DFT spectrum of 4 using UB3LYP/6-31G(d) level of theory with dichloromethane as solvent. 


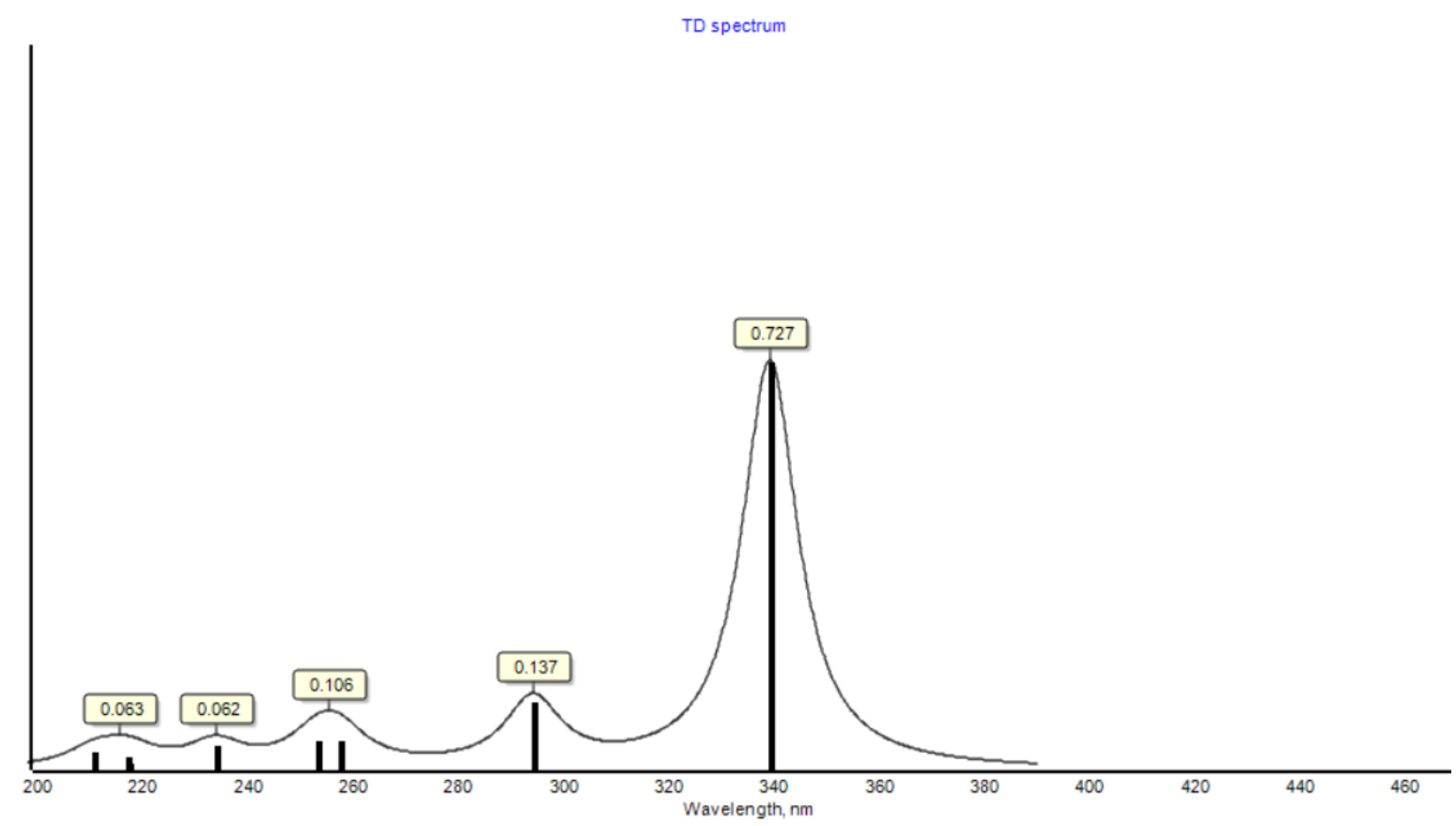

Figure S25. TD-DFT spectrum of 5 using UB3LYP/6-31G(d) level of theory with dichloromethane as solvent.

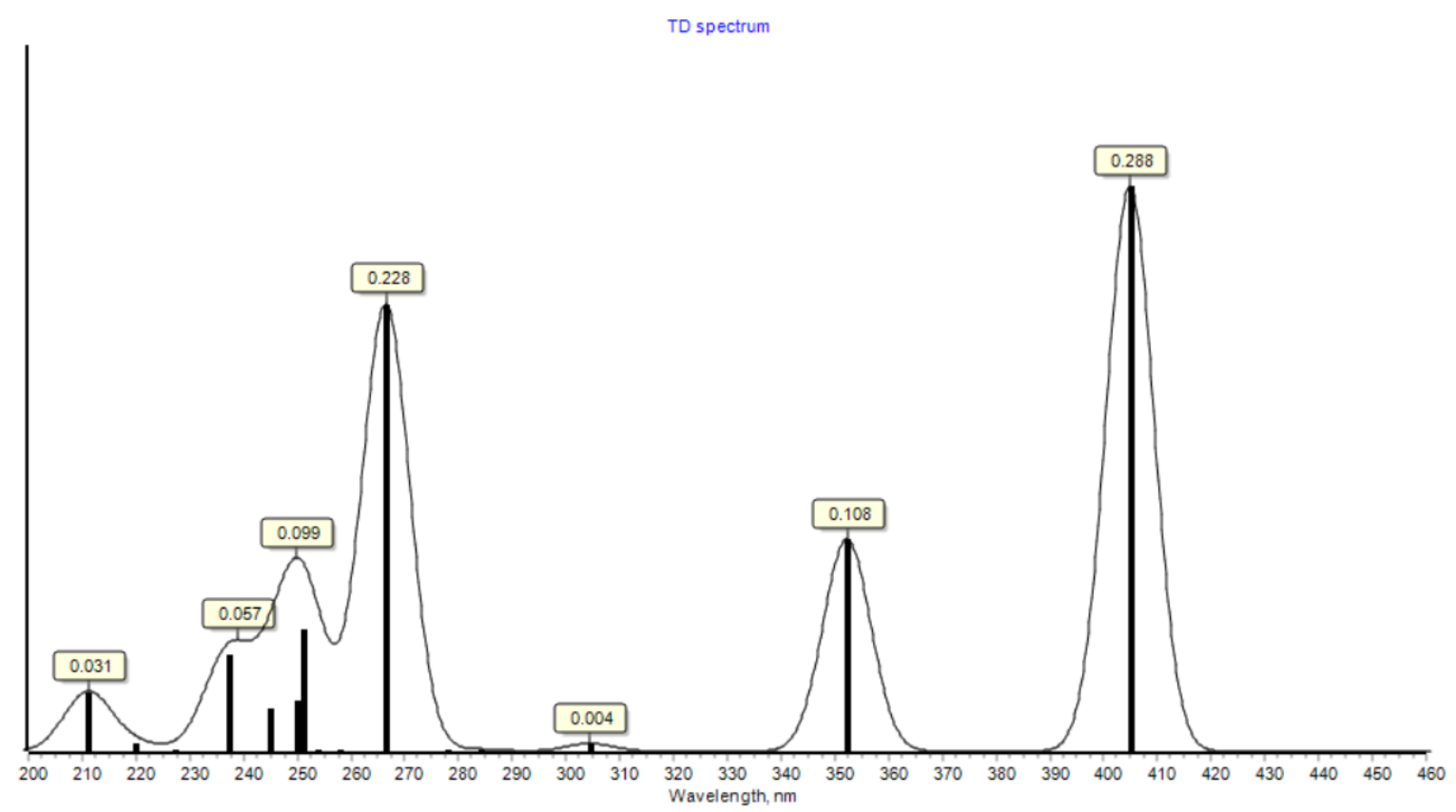

Figure S26. TD-DFT spectrum of 6 using UB3LYP/6-31G(d) level of theory with dichloromethane as solvent. 


\section{Casting and polymer measurements}<smiles></smiles>

\section{1-Me}

Figure S27. The molecular structure of compound 1-Me used in casting and polymer measurements.
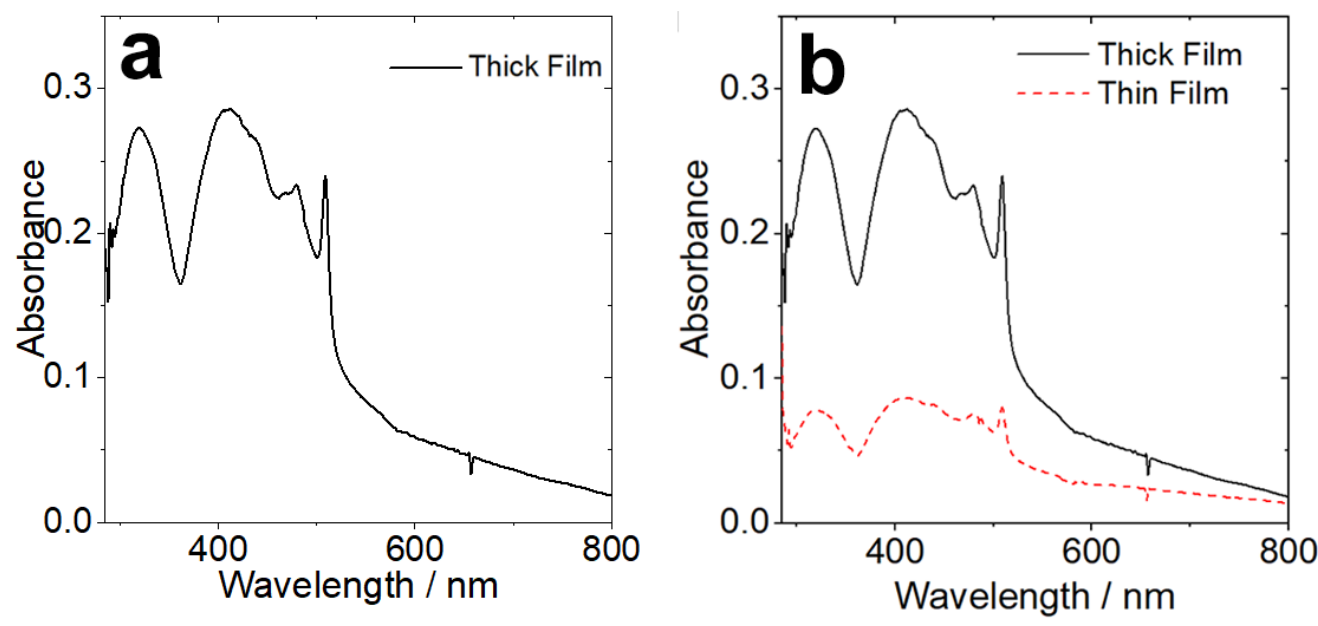

Figure S28. (a) UV-vis absorption of 1-Me casting film on quartz plates. (b) Comparison of the UVVis absorption of the thick film and the thin film. The solvent (toluene) was evaporated at $20^{\circ} \mathrm{C}$.
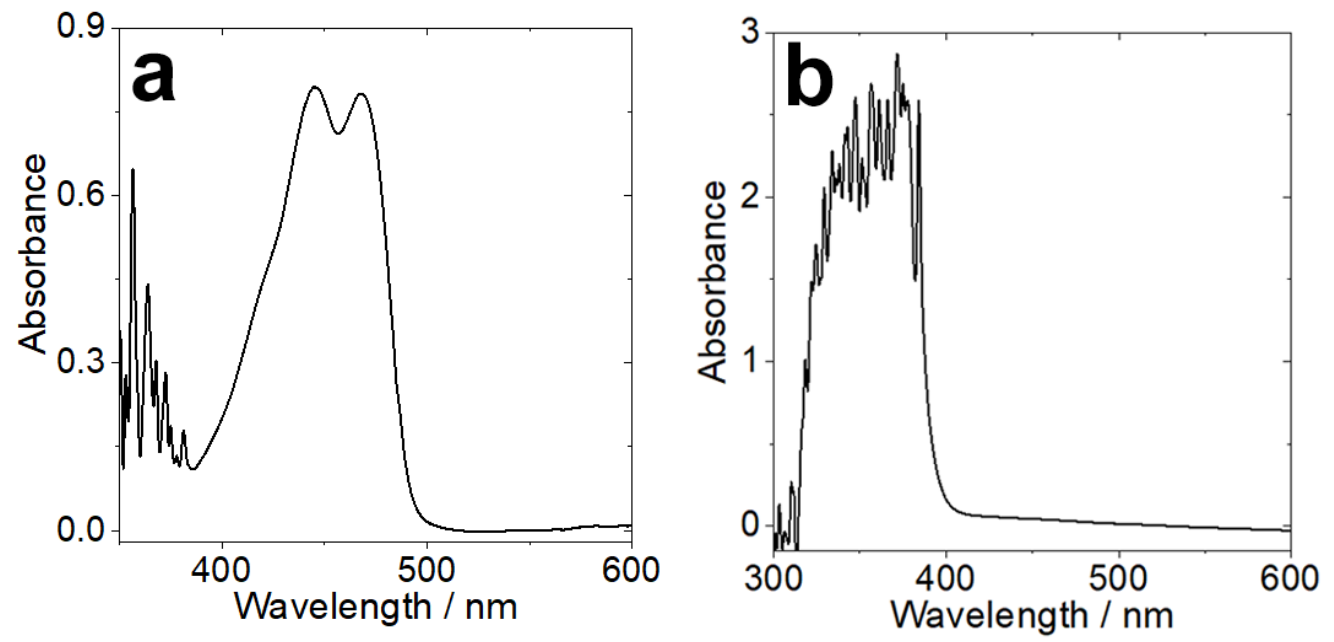

Figure S29. UV-vis absorption of (a) 1-Me doped in the polymer film (Clear Flex $\left.50^{\circledR}, \mathrm{CLRFLX}\right)^{2}$ and (b) blank polymer film at $20^{\circ} \mathrm{C}$. 


\section{Polymer Film Preparation}

Clear Flex 50 is available commercially, which includes two parts (Part A contains 4,4'methylenedicyclohexyl diisocyanate, and part B contains phenylmercury neodecanoate). To prepare the film, part $A$ and part $B$ were mixed by weight with the mix ratio of 1:2 (500 mg: 1000 $\mathrm{mg})$, and then stirred with a vortex mixer to form a clear sticky solution. A sample of 1-Me $(10 \mu \mathrm{L}$, $\mathrm{c}=$ ca.1.45 mM in toluene) and polymer precursor $(150 \mu \mathrm{L})$ were mixed with a vortex mixer. The mixture was dropped on quartz plates (diameter, $17 \mathrm{~mm}$; thickness, $1 \mathrm{~mm}$ ) and the film was kept at room temperature to evaporate the solvent for $48 \mathrm{~h}$ before measurements. 


\section{References}

1. Lv, X.; Li, T.; Wu, Q.; Yu, C.; Jiao, L.; Hao, E., Polybrominated BOPHY Dyes: Synthesis, Reactivity, and Properties. J. Org. Chem. 2018, 83 (3), 1134-1145.

2. Kim, J.-H.; Deng, F.; Castellano, F. N.; Kim, J.-H., High Efficiency Low-Power Upconverting Soft Materials. Chem. Mater. 2012, 24 (12), 2250-2252. 


\section{Cartesian Coordinates}

1, So, UB3LYP/6-31G, E(scf) = -1054.0302353

\begin{tabular}{crrr}
\hline & $\mathbf{x}(\AA)$ & $\mathbf{y}(\AA)$ & \multicolumn{1}{c}{$\mathbf{z}(\AA)$} \\
\hline $\mathrm{N}$ & -3.1227190 & 0.1356020 & -0.5547730 \\
$\mathrm{C}$ & -3.0814600 & 1.4185940 & 0.0122110 \\
$\mathrm{~N}$ & -0.6373470 & 0.2434720 & -0.6314530 \\
$\mathrm{~N}$ & -0.6849420 & 1.5863100 & -0.2001850 \\
$\mathrm{C}$ & -1.8717740 & 2.1017360 & 0.1178680 \\
$\mathrm{C}$ & 1.7040450 & 0.1568360 & -0.0598360 \\
$\mathrm{~N}$ & 1.7648010 & 1.5268940 & 0.2378440 \\
$\mathrm{~B}$ & 0.6326080 & 2.4840840 & -0.1398760 \\
$\mathrm{C}$ & -4.3998850 & -0.3150540 & -0.4954970 \\
$\mathrm{C}$ & 3.0051480 & 1.8054840 & 0.7087210 \\
$\mathrm{C}$ & 3.7720760 & 0.6217600 & 0.7346640 \\
$\mathrm{C}$ & 2.9644590 & -0.4122070 & 0.2473810 \\
$\mathrm{C}$ & 0.5195830 & -0.4090020 & -0.5265280 \\
$\mathrm{~F}$ & 0.8496650 & 3.0775980 & -1.4097440 \\
$\mathrm{~B}$ & -1.9137630 & -0.4664740 & -1.2733590 \\
$\mathrm{~F}$ & -1.7855320 & -1.8603260 & -1.0314170 \\
$\mathrm{~F}$ & -1.9441420 & -0.1981170 & -2.6658210 \\
$\mathrm{C}$ & -4.3929070 & 1.7521560 & 0.4313680 \\
$\mathrm{C}$ & -5.2121100 & 0.6614090 & 0.1183560 \\
$\mathrm{H}$ & -1.8288070 & 3.1145310 & 0.4960240 \\
$\mathrm{H}$ & -4.6668120 & -1.2820630 & -0.8912820 \\
$\mathrm{H}$ & 3.2809960 & 2.8098740 & 0.9882430 \\
$\mathrm{H}$ & 4.7932240 & 0.5416980 & 1.0722910 \\
$\mathrm{H}$ & 3.2291170 & -1.4530870 & 0.1369780 \\
$\mathrm{H}$ & 0.4698390 & -1.4493650 & -0.8193280 \\
$\mathrm{H}$ & -4.6842670 & 2.6719330 & 0.9159000 \\
$\mathrm{H}$ & -6.2700360 & 0.5707160 & 0.3073420 \\
$\mathrm{~F}$ & 0.4180580 & 3.4786470 & 0.8513110 \\
\hline & & &
\end{tabular}

1, $S_{0}$, UB3LYP/6-31G(d), E(scf) = -1054.2853611

\begin{tabular}{rrrr}
\hline & $\mathbf{x}(\mathbf{A})$ & $\mathbf{y}(\AA \mathbf{)})$ & \multicolumn{1}{c}{$\mathbf{z}(\mathbf{\AA})$} \\
\hline $\mathrm{N}$ & -3.1174930 & 0.1248710 & -0.5538610 \\
$\mathrm{C}$ & -3.0727520 & 1.3938900 & 0.0077620 \\
$\mathrm{~N}$ & -0.6352870 & 0.2633940 & -0.6649660 \\
$\mathrm{~N}$ & -0.6818920 & 1.5897550 & -0.2386960 \\
$\mathrm{C}$ & -1.8622460 & 2.0857610 & 0.0901800 \\
$\mathrm{C}$ & 1.6951780 & 0.1800010 & -0.0507180 \\
$\mathrm{~N}$ & 1.7593230 & 1.5354270 & 0.2435770 \\
$\mathrm{~B}$ & 0.6449110 & 2.5089450 & -0.1960050 \\
$\mathrm{C}$ & -4.3820450 & -0.3234120 & -0.4631290 \\
$\mathrm{C}$ & 2.9830610 & 1.7941410 & 0.7373930 \\
$\mathrm{C}$ & 3.7396970 & 0.6099160 & 0.7856560 \\
$\mathrm{C}$ & 2.9339610 & -0.4097630 & 0.2814780 \\
$\mathrm{C}$ & 0.5131810 & -0.3792750 & -0.5412630 \\
$\mathrm{~F}$ & 0.8759720 & 3.0010370 & -1.4576990 \\
$\mathrm{~B}$ & -1.9178690 & -0.4535530 & -1.3341480 \\
$\mathrm{~F}$ & -1.7812640 & -1.8099390 & -1.1350290 \\
$\mathrm{~F}$ & -1.9681960 & -0.1075690 & -2.6627530 \\
$\mathrm{C}$ & -4.3668620 & 1.7290350 & 0.4612900 \\
$\mathrm{C}$ & -5.1869880 & 0.6401340 & 0.1699670 \\
$\mathrm{H}$ & -1.8265210 & 3.1026990 & 0.4627430 \\
$\mathrm{H}$ & -4.6515990 & -1.2937840 & -0.8575220 \\
$\mathrm{H}$ & 3.2610600 & 2.8002960 & 1.0206270 \\
$\mathrm{H}$ & 4.7533150 & 0.5228480 & 1.1516080 \\
$\mathrm{H}$ & 3.1815290 & -1.4580170 & 0.1788090 \\
$\mathrm{H}$ & 0.4715450 & -1.4199710 & -0.8402890 \\
$\mathrm{H}$ & -4.6426180 & 2.6496830 & 0.9582980 \\
$\mathrm{H}$ & -6.2414280 & 0.5385320 & 0.3861980 \\
$\mathrm{~F}$ & 0.4294420 & 3.4985530 & 0.7378970 \\
\hline & & &
\end{tabular}

1, So, UB3LYP/6-31G(d,p), E(scf) = -1054.2983778

\begin{tabular}{rrrr}
\hline & $\mathbf{x}(\AA)$ & \multicolumn{1}{c}{$\mathbf{y}(\AA)$} & \multicolumn{1}{c}{$\mathbf{z}(\AA)$} \\
\hline $\mathrm{N}$ & -3.1168100 & 0.1251330 & -0.5559160 \\
$\mathrm{C}$ & -3.0722280 & 1.3935860 & 0.0067080 \\
$\mathrm{~N}$ & -0.6350180 & 0.2648980 & -0.6669470 \\
$\mathrm{~N}$ & -0.6819570 & 1.5901690 & -0.2390730 \\
$\mathrm{C}$ & -1.8621800 & 2.0860760 & 0.0903740 \\
$\mathrm{C}$ & 1.6944660 & 0.1804510 & -0.0515530 \\
$\mathrm{~N}$ & 1.7584860 & 1.5352390 & 0.2450830 \\
$\mathrm{~B}$ & 0.6439040 & 2.5096400 & -0.1920810 \\
$\mathrm{C}$ & -4.3812770 & -0.3236300 & -0.4649300 \\
$\mathrm{C}$ & 2.9823280 & 1.7931890 & 0.7394010 \\
$\mathrm{C}$ & 3.7384810 & 0.6087970 & 0.7856960 \\
$\mathrm{C}$ & 2.9330000 & -0.4099440 & 0.2799010 \\
$\mathrm{C}$ & 0.5130300 & -0.3785450 & -0.5437270 \\
$\mathrm{~F}$ & 0.8764280 & 3.0066500 & -1.4516330 \\
$\mathrm{~B}$ & -1.9167740 & -0.4524460 & -1.3362670 \\
$\mathrm{~F}$ & -1.7774730 & -1.8085950 & -1.1373680 \\
$\mathrm{~F}$ & -1.9677700 & -0.1063210 & -2.6648700 \\
$\mathrm{C}$ & -4.3659790 & 1.7278320 & 0.4615890 \\
$\mathrm{C}$ & -5.1856770 & 0.6390840 & 0.1697450 \\
$\mathrm{H}$ & -1.8252290 & 3.1021470 & 0.4649580 \\
$\mathrm{H}$ & -4.6521590 & -1.2928400 & -0.8595840 \\
$\mathrm{H}$ & 3.2615400 & 2.7977920 & 1.0245710 \\
$\mathrm{H}$ & 4.7513350 & 0.5213030 & 1.1513690 \\
$\mathrm{H}$ & 3.1800120 & -1.4573790 & 0.1754000 \\
$\mathrm{H}$ & 0.4698840 & -1.4188510 & -0.8436100 \\
$\mathrm{H}$ & -4.6411630 & 2.6475510 & 0.9590130 \\
$\mathrm{H}$ & -6.2391830 & 0.5368110 & 0.3863180 \\
$\mathrm{~F}$ & 0.4251000 & 3.4958390 & 0.7448390 \\
\hline & & &
\end{tabular}

$1, S_{0}$, UB3LYP/6-31+G(d,p), E(scf) = -1054.3506970

\begin{tabular}{rrrr}
\hline & \multicolumn{1}{c}{$\mathbf{x ( \AA )}$} & \multicolumn{1}{c}{$\mathbf{y}(\AA)$} & \multicolumn{1}{c}{$\mathbf{z}(\AA)$} \\
\hline $\mathrm{N}$ & -3.1304360 & 0.1186500 & -0.5418890 \\
$\mathrm{C}$ & -3.0861520 & 1.3930580 & 0.0076530 \\
$\mathrm{~N}$ & -0.6358460 & 0.2476360 & -0.6173130 \\
$\mathrm{~N}$ & -0.6880590 & 1.5746580 & -0.1908640 \\
$\mathrm{C}$ & -1.8736470 & 2.0792120 & 0.1105930 \\
$\mathrm{C}$ & 1.7083360 & 0.1802400 & -0.0485250 \\
$\mathrm{~N}$ & 1.7699980 & 1.5327760 & 0.2597200 \\
$\mathrm{~B}$ & 0.6342800 & 2.4961370 & -0.1283050 \\
$\mathrm{C}$ & -4.4056230 & -0.3123550 & -0.4894740 \\
$\mathrm{C}$ & 3.0099140 & 1.7993310 & 0.7137490 \\
$\mathrm{C}$ & 3.7774540 & 0.6201640 & 0.7242120 \\
$\mathrm{C}$ & 2.9633860 & -0.4026850 & 0.2354800 \\
$\mathrm{C}$ & 0.5216960 & -0.3860960 & -0.5201960 \\
$\mathrm{~F}$ & 0.8416260 & 3.0423040 & -1.3840730 \\
$\mathrm{~B}$ & -1.9163830 & -0.4827890 & -1.2716010 \\
$\mathrm{~F}$ & -1.7889780 & -1.8433140 & -1.0355290 \\
$\mathrm{~F}$ & -1.9409660 & -0.1854120 & -2.6240930 \\
$\mathrm{C}$ & -4.3899050 & 1.7509920 & 0.4171660 \\
$\mathrm{C}$ & -5.2162750 & 0.6691040 & 0.1098690 \\
$\mathrm{H}$ & -1.8465320 & 3.0996690 & 0.4752320 \\
$\mathrm{H}$ & -4.6779950 & -1.2816520 & -0.8835880 \\
$\mathrm{H}$ & 3.2905580 & 2.8042040 & 0.9975630 \\
$\mathrm{H}$ & 4.8034980 & 0.5388530 & 1.0533190 \\
$\mathrm{H}$ & 3.2170120 & -1.4466080 & 0.1111530 \\
$\mathrm{H}$ & 0.4901650 & -1.4251070 & -0.8275340 \\
$\mathrm{H}$ & -4.6686650 & 2.6805780 & 0.8943490 \\
$\mathrm{H}$ & -6.2780650 & 0.5845790 & 0.2919130 \\
$\mathrm{~F}$ & 0.4227210 & 3.4675100 & 0.8384180 \\
\hline & & &
\end{tabular}

1, So, UB3LYP/cc-pVTZ, E(scf) $=-1054.6778918$

\begin{tabular}{cccr}
\hline & $\mathbf{x}(\AA)$ & $\mathbf{y}(\AA)$ & \multicolumn{1}{c}{$\mathbf{z}(\AA)$} \\
\hline $\mathrm{N}$ & -3.1281290 & 0.1187510 & -0.5442610 \\
$\mathrm{C}$ & -3.0801790 & 1.3877150 & 0.0044450 \\
$\mathrm{~N}$ & -0.6331010 & 0.2493250 & -0.6141260 \\
\hline
\end{tabular}




\begin{tabular}{rrrr}
\hline N & -0.6914580 & 1.5717940 & -0.1873640 \\
$\mathrm{C}$ & -1.8722710 & 2.0703360 & 0.1092460 \\
$\mathrm{C}$ & 1.7025420 & 0.1868800 & -0.0506290 \\
$\mathrm{~N}$ & 1.7679160 & 1.5334800 & 0.2593750 \\
$\mathrm{~B}$ & 0.6313840 & 2.5025960 & -0.1216660 \\
$\mathrm{C}$ & -4.3986520 & -0.3078320 & -0.4885740 \\
$\mathrm{C}$ & 3.0032800 & 1.7936750 & 0.7127030 \\
$\mathrm{C}$ & 3.7644280 & 0.6179980 & 0.7187140 \\
$\mathrm{C}$ & 2.9508760 & -0.3966010 & 0.2297280 \\
$\mathrm{C}$ & 0.5201440 & -0.3766140 & -0.5197660 \\
$\mathrm{~F}$ & 0.8331170 & 3.0486880 & -1.3676680 \\
$\mathrm{~B}$ & -1.9139240 & -0.4879830 & -1.2745910 \\
$\mathrm{~F}$ & -1.7873830 & -1.8381290 & -1.0369930 \\
$\mathrm{~F}$ & -1.9342560 & -0.1899160 & -2.6170800 \\
$\mathrm{C}$ & -4.3768210 & 1.7459630 & 0.4140170 \\
$\mathrm{C}$ & -5.2025180 & 0.6708500 & 0.1094060 \\
$\mathrm{H}$ & -1.8484430 & 3.0863640 & 0.4746520 \\
$\mathrm{H}$ & -4.6740650 & -1.2727240 & -0.8800720 \\
$\mathrm{H}$ & 3.2868390 & 2.7925420 & 0.9991170 \\
$\mathrm{H}$ & 4.7862170 & 0.5333430 & 1.0456710 \\
$\mathrm{H}$ & 3.2007950 & -1.4364750 & 0.1024730 \\
$\mathrm{H}$ & 0.4917890 & -1.4117010 & -0.8265930 \\
$\mathrm{H}$ & -4.6517270 & 2.6722770 & 0.8897730 \\
$\mathrm{H}$ & -6.2597780 & 0.5889060 & 0.2927160 \\
$\mathrm{~F}$ & 0.4204950 & 3.4601340 & 0.8447480 \\
\hline
\end{tabular}

1, So, UM06-2X/6-31G, E(scf) $=-1053.6610222$

\begin{tabular}{rrrr}
\hline & \multicolumn{1}{c}{$\mathbf{x}(\AA \mathbf{)})$} & \multicolumn{1}{c}{$\mathbf{y}(\AA \mathbf{)})$} & \multicolumn{1}{c}{$\mathbf{z ( \AA ̊ )}$} \\
\hline $\mathrm{N}$ & -3.1064600 & 0.1446240 & -0.5588280 \\
$\mathrm{C}$ & -3.0648130 & 1.4173110 & 0.0110110 \\
$\mathrm{~N}$ & -0.6354500 & 0.2579030 & -0.6594870 \\
$\mathrm{~N}$ & -0.6830300 & 1.5910810 & -0.2294390 \\
$\mathrm{C}$ & -1.8561160 & 2.1056870 & 0.1021040 \\
$\mathrm{C}$ & 1.6869280 & 0.1592510 & -0.0608680 \\
$\mathrm{~N}$ & 1.7494290 & 1.5221670 & 0.2298830 \\
$\mathrm{~B}$ & 0.6310730 & 2.4803360 & -0.1701550 \\
$\mathrm{C}$ & -4.3696910 & -0.3191680 & -0.4814330 \\
$\mathrm{C}$ & 2.9738790 & 1.8005490 & 0.7202310 \\
$\mathrm{C}$ & 3.7340170 & 0.6168160 & 0.7676070 \\
$\mathrm{C}$ & 2.9325420 & -0.4131740 & 0.2711370 \\
$\mathrm{C}$ & 0.5059990 & -0.4014190 & -0.5444930 \\
$\mathrm{~F}$ & 0.8620240 & 3.0534870 & -1.4391010 \\
$\mathrm{~B}$ & -1.9080200 & -0.4431330 & -1.2985180 \\
$\mathrm{~F}$ & -1.7704000 & -1.8324880 & -1.0731860 \\
$\mathrm{~F}$ & -1.9530430 & -0.1567460 & -2.6797680 \\
$\mathrm{C}$ & -4.3650210 & 1.7370710 & 0.4546950 \\
$\mathrm{C}$ & -5.1786100 & 0.6433830 & 0.1511170 \\
$\mathrm{H}$ & -1.8089700 & 3.1195310 & 0.4803820 \\
$\mathrm{H}$ & -4.6295640 & -1.2869090 & -0.8793300 \\
$\mathrm{H}$ & 3.2434830 & 2.8068200 & 0.9979160 \\
$\mathrm{H}$ & 4.7464830 & 0.5338260 & 1.1247980 \\
$\mathrm{H}$ & 3.1929360 & -1.4550460 & 0.1702230 \\
$\mathrm{H}$ & 0.4534410 & -1.4418060 & -0.8408730 \\
$\mathrm{H}$ & -4.6542690 & 2.6510330 & 0.9491660 \\
$\mathrm{H}$ & -6.2306170 & 0.5424890 & 0.3577370 \\
$\mathrm{~F}$ & 0.4089580 & 3.4801640 & 0.8048720 \\
\hline & & & \\
& & &
\end{tabular}

1, $\mathrm{S}_{0}, \mathrm{UM06-2X/6-31G(d),} \mathrm{E(scf)} \mathrm{=} \mathrm{-1053.9123919}$

\begin{tabular}{cccr}
\hline & $\mathbf{x}(\AA)$ & $\mathbf{y}(\AA)$ & \multicolumn{1}{c}{$\mathbf{z}(\AA)$} \\
\hline $\mathrm{N}$ & -3.0967370 & 0.1266120 & -0.5549820 \\
$\mathrm{C}$ & -3.0503810 & 1.3853240 & 0.0112130 \\
$\mathrm{~N}$ & -0.6327370 & 0.2829460 & -0.7155750 \\
$\mathrm{~N}$ & -0.6770690 & 1.6026090 & -0.2908600 \\
$\mathrm{C}$ & -1.8424950 & 2.0906220 & 0.0634250 \\
$\mathrm{C}$ & 1.6718630 & 0.1851880 & -0.0448400 \\
$\mathrm{~N}$ & 1.7402300 & 1.5353880 & 0.2368450 \\
\hline
\end{tabular}

\begin{tabular}{rrrr}
\hline B & 0.6538540 & 2.5105790 & -0.2627760 \\
C & -4.3450530 & -0.3387410 & -0.4311590 \\
C & 2.9435620 & 1.7890540 & 0.7643280 \\
C & 3.6845030 & 0.6007480 & 0.8517230 \\
C & 2.8860630 & -0.4135820 & 0.3325700 \\
C & 0.4969410 & -0.3677190 & -0.5671520 \\
F & 0.9132970 & 2.9348780 & -1.5348320 \\
B & -1.9178310 & -0.4132300 & -1.3917970 \\
F & -1.7638880 & -1.7676980 & -1.2526300 \\
F & -2.0011010 & 0.0026100 & -2.6903420 \\
C & -4.3267010 & 1.7006170 & 0.5082410 \\
C & -5.1406100 & 0.6055360 & 0.2352630 \\
H & -1.8044500 & 3.1098840 & 0.4331810 \\
H & -4.6101930 & -1.3093290 & -0.8282650 \\
H & 3.2182770 & 2.7975620 & 1.0424340 \\
H & 4.6829710 & 0.5066510 & 1.2524350 \\
H & 3.1234220 & -1.4653340 & 0.2489360 \\
H & 0.4541510 & -1.4087560 & -0.8694290 \\
H & -4.5959910 & 2.6131580 & 1.0222080 \\
H & -6.1850640 & 0.4884900 & 0.4838180 \\
F & 0.4282870 & 3.5295700 & 0.6254230 \\
\hline & & &
\end{tabular}

1, So, UM06-2X/6-31G(d,p), E(scf) = -1053.9233088

\begin{tabular}{rrrr}
\hline & \multicolumn{1}{c}{$\mathbf{x ( \AA )}$} & \multicolumn{1}{c}{$\mathbf{y}(\AA)$} & \multicolumn{1}{c}{$\mathbf{z}(\AA) \mathbf{)}$} \\
\hline $\mathrm{N}$ & -3.0961720 & 0.1265880 & -0.5550750 \\
$\mathrm{C}$ & -3.0499550 & 1.3855050 & 0.0101830 \\
$\mathrm{~N}$ & -0.6325840 & 0.2834900 & -0.7162530 \\
$\mathrm{~N}$ & -0.6771820 & 1.6027590 & -0.2919330 \\
$\mathrm{C}$ & -1.8423240 & 2.0914510 & 0.0622840 \\
$\mathrm{C}$ & 1.6714100 & 0.1856070 & -0.0453280 \\
$\mathrm{~N}$ & 1.7395800 & 1.5355220 & 0.2367840 \\
$\mathrm{~B}$ & 0.6531550 & 2.5107870 & -0.2621700 \\
$\mathrm{C}$ & -4.3444700 & -0.3390130 & -0.4306470 \\
$\mathrm{C}$ & 2.9429320 & 1.7891860 & 0.7645760 \\
$\mathrm{C}$ & 3.6835790 & 0.6008150 & 0.8515710 \\
$\mathrm{C}$ & 2.8853380 & -0.4134020 & 0.3320120 \\
$\mathrm{C}$ & 0.4967740 & -0.3677770 & -0.5680230 \\
$\mathrm{~F}$ & 0.9135270 & 2.9371120 & -1.5333930 \\
$\mathrm{~B}$ & -1.9171360 & -0.4141460 & -1.3909120 \\
$\mathrm{~F}$ & -1.7610490 & -1.7683890 & -1.2491240 \\
$\mathrm{~F}$ & -2.0011940 & -0.0014660 & -2.6904290 \\
$\mathrm{C}$ & -4.3259660 & 1.7010590 & 0.5074340 \\
$\mathrm{C}$ & -5.1396020 & 0.6055640 & 0.2356170 \\
$\mathrm{H}$ & -1.8027380 & 3.1106430 & 0.4321500 \\
$\mathrm{H}$ & -4.6105830 & -1.3093180 & -0.8265100 \\
$\mathrm{H}$ & 3.2184880 & 2.7967940 & 1.0433410 \\
$\mathrm{H}$ & 4.6814910 & 0.5067610 & 1.2519920 \\
$\mathrm{H}$ & 3.1222660 & -1.4645740 & 0.2475800 \\
$\mathrm{H}$ & 0.4522100 & -1.4088560 & -0.8700070 \\
$\mathrm{H}$ & -4.5947360 & 2.6137450 & 1.0201820 \\
$\mathrm{H}$ & -6.1832900 & 0.4884240 & 0.4845270 \\
$\mathrm{~F}$ & 0.4253480 & 3.5287670 & 0.6269740 \\
\hline & & & \\
& & &
\end{tabular}

1, So, UM06-2X/6-31+G(d,p), E(scf) = -1053.9642515

\begin{tabular}{crrr}
\hline & $\mathbf{x}(\AA)$ & $\mathbf{y}(\AA)$ & \multicolumn{1}{c}{$\mathbf{z}(\AA)$} \\
\hline $\mathrm{N}$ & -3.1023550 & 0.1193250 & -0.5447890 \\
$\mathrm{C}$ & -3.0558940 & 1.3807500 & 0.0157780 \\
$\mathrm{~N}$ & -0.6320470 & 0.2770810 & -0.6987540 \\
$\mathrm{~N}$ & -0.6800110 & 1.5974000 & -0.2745640 \\
$\mathrm{C}$ & -1.8480460 & 2.0875670 & 0.0716930 \\
$\mathrm{C}$ & 1.6767850 & 0.1860230 & -0.0379480 \\
$\mathrm{~N}$ & 1.7445430 & 1.5355140 & 0.2481770 \\
$\mathrm{~B}$ & 0.6503130 & 2.5022210 & -0.2424350 \\
$\mathrm{C}$ & -4.3572660 & -0.3381100 & -0.4376300 \\
$\mathrm{C}$ & 2.9568080 & 1.7926660 & 0.7582750 \\
$\mathrm{C}$ & 3.7018300 & 0.6047680 & 0.8324680 \\
\hline
\end{tabular}




\begin{tabular}{rrrr}
\hline C & 2.8985410 & -0.4113880 & 0.3200620 \\
C & 0.5013810 & -0.3706700 & -0.5574880 \\
F & 0.9059330 & 2.9326720 & -1.5214450 \\
B & -1.9175450 & -0.4196820 & -1.3685710 \\
F & -1.7641360 & -1.7808530 & -1.2341610 \\
F & -1.9972030 & -0.0055750 & -2.6760640 \\
C & -4.3372490 & 1.7073750 & 0.4947230 \\
C & -5.1551370 & 0.6145820 & 0.2161070 \\
H & -1.8167670 & 3.1096870 & 0.4359590 \\
H & -4.6251720 & -1.3075830 & -0.8354720 \\
H & 3.2344710 & 2.8008570 & 1.0344500 \\
H & 4.7060430 & 0.5127490 & 1.2183040 \\
H & 3.1378550 & -1.4618410 & 0.2283690 \\
H & 0.4660540 & -1.4113580 & -0.8640050 \\
H & -4.6075110 & 2.6244550 & 0.9996780 \\
H & -6.2033000 & 0.5051890 & 0.4510200 \\
F & 0.4262020 & 3.5298160 & 0.6456650 \\
\hline & & & \\
\hline
\end{tabular}

1, So, UM06-2X/cc-pVTZ, E(scf) = -1054.3246969

\begin{tabular}{rrrr}
\hline & \multicolumn{1}{c}{$\mathbf{x}(\AA)$} & \multicolumn{1}{c}{$\mathbf{y}(\AA)$} & \multicolumn{1}{c}{$\mathbf{z}(\AA)$} \\
\hline $\mathrm{N}$ & -3.1036780 & 0.1210960 & -0.5456290 \\
$\mathrm{C}$ & -3.0564530 & 1.3806740 & 0.0104800 \\
$\mathrm{~N}$ & -0.6321740 & 0.2693840 & -0.6715870 \\
$\mathrm{~N}$ & -0.6843840 & 1.5885690 & -0.2483900 \\
$\mathrm{C}$ & -1.8509780 & 2.0793290 & 0.0793190 \\
$\mathrm{C}$ & 1.6768260 & 0.1889540 & -0.0395540 \\
$\mathrm{~N}$ & 1.7437420 & 1.5334080 & 0.2537960 \\
$\mathrm{~B}$ & 0.6422800 & 2.5016240 & -0.2113790 \\
$\mathrm{C}$ & -4.3585800 & -0.3264730 & -0.4489840 \\
$\mathrm{C}$ & 2.9585010 & 1.7888200 & 0.7474850 \\
$\mathrm{C}$ & 3.7056690 & 0.6076850 & 0.8016580 \\
$\mathrm{C}$ & 2.9007450 & -0.4036810 & 0.2951870 \\
$\mathrm{C}$ & 0.5021800 & -0.3679720 & -0.5455090 \\
$\mathrm{~F}$ & 0.8822820 & 2.9530810 & -1.4781000 \\
$\mathrm{~B}$ & -1.9131260 & -0.4367030 & -1.3446200 \\
$\mathrm{~F}$ & -1.7702550 & -1.7885220 & -1.1858190 \\
$\mathrm{~F}$ & -1.9705480 & -0.0510730 & -2.6539400 \\
$\mathrm{C}$ & -4.3358460 & 1.7147040 & 0.4719120 \\
$\mathrm{C}$ & -5.1548700 & 0.6298190 & 0.1894520 \\
$\mathrm{H}$ & -1.8235500 & 3.0981850 & 0.4414270 \\
$\mathrm{H}$ & -4.6271880 & -1.2928710 & -0.8437660 \\
$\mathrm{H}$ & 3.2362800 & 2.7923570 & 1.0263260 \\
$\mathrm{H}$ & 4.7121730 & 0.5168480 & 1.1704920 \\
$\mathrm{H}$ & 3.1405010 & -1.4488150 & 0.1915090 \\
$\mathrm{H}$ & 0.4711880 & -1.4045260 & -0.8529250 \\
$\mathrm{H}$ & -4.6039800 & 2.6323460 & 0.9684220 \\
$\mathrm{H}$ & -6.2023850 & 0.5278830 & 0.4123130 \\
$\mathrm{~F}$ & 0.4227480 & 3.5095090 & 0.6878270 \\
\hline & & & \\
& & &
\end{tabular}

1, $S_{0}, U \omega B 97 X D / 6-31 G, E(s c f)=-1053.7063093$

\begin{tabular}{rrrr}
\hline & \multicolumn{1}{c}{$\mathbf{x ( \AA )}$} & \multicolumn{1}{c}{$\mathbf{y}(\AA \mathbf{)})$} & \multicolumn{1}{c}{$\mathbf{z ( \AA ̊ )}$} \\
\hline $\mathrm{N}$ & -3.1114330 & 0.1413870 & -0.5586480 \\
$\mathrm{C}$ & -3.0679110 & 1.4104650 & 0.0201250 \\
$\mathrm{~N}$ & -0.6374880 & 0.2488620 & -0.6368990 \\
$\mathrm{~N}$ & -0.6840420 & 1.5858130 & -0.2064390 \\
$\mathrm{C}$ & -1.8603540 & 2.0957630 & 0.1215990 \\
$\mathrm{C}$ & 1.6889910 & 0.1601170 & -0.0506390 \\
$\mathrm{~N}$ & 1.7544880 & 1.5250060 & 0.2321110 \\
$\mathrm{~B}$ & 0.6290330 & 2.4775660 & -0.1569640 \\
$\mathrm{C}$ & -4.3803660 & -0.3112500 & -0.4996760 \\
$\mathrm{C}$ & 2.9870650 & 1.8042060 & 0.7034190 \\
$\mathrm{C}$ & 3.7472060 & 0.6222090 & 0.7457470 \\
$\mathrm{C}$ & 2.9387210 & -0.4108480 & 0.2659500 \\
$\mathrm{C}$ & 0.5075430 & -0.4045420 & -0.5229260 \\
$\mathrm{~F}$ & 0.8475940 & 3.0562630 & -1.4325960 \\
$\mathrm{~B}$ & -1.9077200 & -0.4491910 & -1.2845760 \\
\hline
\end{tabular}

\begin{tabular}{rrrr}
\hline F & -1.7783250 & -1.8457750 & -1.0588390 \\
F & -1.9398170 & -0.1642530 & -2.6729500 \\
C & -4.3706440 & 1.7378580 & 0.4496650 \\
C & -5.1888860 & 0.6522890 & 0.1286360 \\
H & -1.8197530 & 3.1080000 & 0.5012710 \\
H & -4.6452050 & -1.2727080 & -0.9080910 \\
H & 3.2627490 & 2.8110100 & 0.9712160 \\
H & 4.7656610 & 0.5405910 & 1.0879640 \\
H & 3.1987530 & -1.4532070 & 0.1650160 \\
H & 0.4604520 & -1.4449120 & -0.8157500 \\
H & -4.6599740 & 2.6516640 & 0.9452140 \\
H & -6.2449160 & 0.5582170 & 0.3207230 \\
F & 0.4156990 & 3.4830400 & 0.8237410 \\
\hline & & & \\
\hline
\end{tabular}

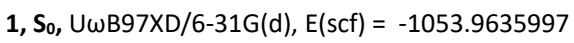

\begin{tabular}{rrrr}
\hline & \multicolumn{1}{c}{$\mathbf{x ( \AA )}$} & \multicolumn{1}{c}{$\mathbf{y}(\AA)$} & \multicolumn{1}{c}{$\mathbf{z}(\AA)$} \\
\hline N & -3.1037480 & 0.1297400 & -0.5619410 \\
C & -3.0564100 & 1.3837190 & 0.0169700 \\
N & -0.6357240 & 0.2714060 & -0.6825270 \\
N & -0.6796600 & 1.5950050 & -0.2553940 \\
C & -1.8491060 & 2.0811260 & 0.0905470 \\
C & 1.6762480 & 0.1849420 & -0.0390350 \\
N & 1.7465240 & 1.5368580 & 0.2382180 \\
B & 0.6445380 & 2.5061040 & -0.2268000 \\
C & -4.3592110 & -0.3234810 & -0.4636830 \\
C & 2.9617780 & 1.7926070 & 0.7384290 \\
C & 3.7066280 & 0.6080260 & 0.8102630 \\
C & 2.8995990 & -0.4093300 & 0.3110650 \\
C & 0.4993880 & -0.3725580 & -0.5417380 \\
F & 0.8841460 & 2.9682600 & -1.4962560 \\
B & -1.9127520 & -0.4280980 & -1.3616430 \\
F & -1.7735450 & -1.7875740 & -1.1930090 \\
F & -1.9699200 & -0.0497330 & -2.6792980 \\
C & -4.3382750 & 1.7064680 & 0.4923880 \\
C & -5.1570200 & 0.6218120 & 0.1944490 \\
H & -1.8157130 & 3.0967690 & 0.4670560 \\
H & -4.6284370 & -1.2869710 & -0.8732240 \\
H & 3.2424820 & 2.8009170 & 1.0088710 \\
H & 4.7146230 & 0.5167220 & 1.1882560 \\
H & 3.1392900 & -1.4601260 & 0.2213930 \\
H & 0.4611830 & -1.4137850 & -0.8395060 \\
H & -4.6102480 & 2.6182660 & 1.0065580 \\
H & -6.2077650 & 0.5128950 & 0.4208350 \\
F & 0.4282260 & 3.5136510 & 0.6861600 \\
\hline & & &
\end{tabular}

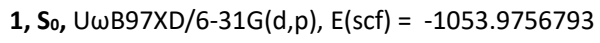

\begin{tabular}{cccc}
\hline & $\mathbf{x}(\mathbf{A})$ & $\mathbf{y}(\AA)$ & \multicolumn{1}{c}{$\mathbf{z}(\AA)$} \\
\hline N & -3.1028900 & 0.1298290 & -0.5616730 \\
$\mathrm{C}$ & -3.0553790 & 1.3837880 & 0.0169630 \\
$\mathrm{~N}$ & -0.6355270 & 0.2722050 & -0.6843500 \\
$\mathrm{~N}$ & -0.6794500 & 1.5952330 & -0.2573790 \\
$\mathrm{C}$ & -1.8482820 & 2.0817620 & 0.0898450 \\
$\mathrm{C}$ & 1.6755900 & 0.1854760 & -0.0401840 \\
$\mathrm{~N}$ & 1.7460350 & 1.5372000 & 0.2374170 \\
$\mathrm{~B}$ & 0.6440600 & 2.5066060 & -0.2275120 \\
$\mathrm{C}$ & -4.3586060 & -0.3231530 & -0.4634210 \\
$\mathrm{C}$ & 2.9610620 & 1.7924060 & 0.7387660 \\
$\mathrm{C}$ & 3.7049320 & 0.6074660 & 0.8111270 \\
$\mathrm{C}$ & 2.8980360 & -0.4094180 & 0.3112710 \\
$\mathrm{C}$ & 0.4990920 & -0.3724380 & -0.5433850 \\
$\mathrm{~F}$ & 0.8848340 & 2.9698490 & -1.4962860 \\
$\mathrm{~B}$ & -1.9122830 & -0.4287870 & -1.3614330 \\
$\mathrm{~F}$ & -1.7702750 & -1.7878610 & -1.1903090 \\
$\mathrm{~F}$ & -1.9709210 & -0.0530430 & -2.6796580 \\
$\mathrm{C}$ & -4.3368090 & 1.7066380 & 0.4929180 \\
$\mathrm{C}$ & -5.1556530 & 0.6222450 & 0.1950420 \\
\hline & & &
\end{tabular}




\begin{tabular}{rrrr}
\hline $\mathrm{H}$ & -1.8125330 & 3.0969260 & 0.4673090 \\
$\mathrm{H}$ & -4.6291980 & -1.2858110 & -0.8725240 \\
$\mathrm{H}$ & 3.2430340 & 2.7996340 & 1.0096820 \\
$\mathrm{H}$ & 4.7117510 & 0.5158070 & 1.1899810 \\
$\mathrm{H}$ & 3.1365860 & -1.4596950 & 0.2211240 \\
$\mathrm{H}$ & 0.4584610 & -1.4138850 & -0.8399760 \\
$\mathrm{H}$ & -4.6078020 & 2.6180970 & 1.0067060 \\
$\mathrm{H}$ & -6.2056310 & 0.5133410 & 0.4213910 \\
$\mathrm{~F}$ & 0.4248890 & 3.5132210 & 0.6859510 \\
\hline
\end{tabular}

1, $S_{0}, U \omega B 97 X D / 6-31+G(d, p), E(s c f)=-1054.0174787$

\begin{tabular}{rrrr}
\hline & $\mathbf{x}(\AA)$ & $\mathbf{y}(\AA)$ & $\mathbf{z}(\AA)$ \\
\hline $\mathrm{N}$ & -3.1130510 & 0.1234860 & -0.5485680 \\
$\mathrm{C}$ & -3.0661590 & 1.3825520 & 0.0196930 \\
$\mathrm{~N}$ & -0.6362100 & 0.2590550 & -0.6464850 \\
$\mathrm{~N}$ & -0.6840910 & 1.5837450 & -0.2211400 \\
$\mathrm{C}$ & -1.8574400 & 2.0767000 & 0.1054570 \\
$\mathrm{C}$ & 1.6855990 & 0.1840310 & -0.0337650 \\
$\mathrm{~N}$ & 1.7538890 & 1.5344730 & 0.2519250 \\
$\mathrm{~B}$ & 0.6367650 & 2.4941300 & -0.1818370 \\
$\mathrm{C}$ & -4.3777400 & -0.3150650 & -0.4800340 \\
$\mathrm{C}$ & 2.9823910 & 1.7963860 & 0.7207290 \\
$\mathrm{C}$ & 3.7357680 & 0.6151150 & 0.7658390 \\
$\mathrm{C}$ & 2.9219080 & -0.4052330 & 0.2800320 \\
$\mathrm{C}$ & 0.5055610 & -0.3790380 & -0.5235110 \\
$\mathrm{~F}$ & 0.8618010 & 2.9869250 & -1.4525120 \\
$\mathrm{~B}$ & -1.9114960 & -0.4487150 & -1.3132760 \\
$\mathrm{~F}$ & -1.7772940 & -1.8136860 & -1.1231880 \\
$\mathrm{~F}$ & -1.9525780 & -0.1009090 & -2.6494680 \\
$\mathrm{C}$ & -4.3561520 & 1.7246480 & 0.4602740 \\
$\mathrm{C}$ & -5.1802570 & 0.6453270 & 0.1513030 \\
$\mathrm{H}$ & -1.8304350 & 3.0959920 & 0.4742350 \\
$\mathrm{H}$ & -4.6492620 & -1.2779380 & -0.8890240 \\
$\mathrm{H}$ & 3.2653260 & 2.8044030 & 0.9892580 \\
$\mathrm{H}$ & 4.7539750 & 0.5283960 & 1.1150970 \\
$\mathrm{H}$ & 3.1658530 & -1.4531690 & 0.1747970 \\
$\mathrm{H}$ & 0.4743570 & -1.4197100 & -0.8262370 \\
$\mathrm{H}$ & -4.6300850 & 2.6443340 & 0.9582040 \\
$\mathrm{H}$ & -6.2370930 & 0.5500570 & 0.3517010 \\
$\mathrm{~F}$ & 0.4232700 & 3.4973470 & 0.7479040 \\
\hline & & & \\
& & & \\
& & \\
& & \\
& & \\
& &
\end{tabular}

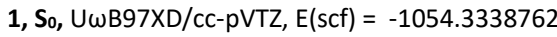

\begin{tabular}{rrrr}
\hline & $\mathbf{x}(\AA)$ & $\mathbf{y}(\AA)$ & \multicolumn{1}{c}{$\mathbf{z}(\AA)$} \\
\hline $\mathrm{N}$ & -3.1136310 & 0.1235470 & -0.5483670 \\
$\mathrm{C}$ & -3.0642760 & 1.3793210 & 0.0140520 \\
$\mathrm{~N}$ & -0.6345200 & 0.2557320 & -0.6293050 \\
$\mathrm{~N}$ & -0.6883490 & 1.5771140 & -0.2060010 \\
$\mathrm{C}$ & -1.8583660 & 2.0675000 & 0.1064710 \\
$\mathrm{C}$ & 1.6836510 & 0.1901500 & -0.0347940 \\
$\mathrm{~N}$ & 1.7542400 & 1.5343630 & 0.2532440 \\
$\mathrm{~B}$ & 0.6321810 & 2.4990180 & -0.1633760 \\
$\mathrm{C}$ & -4.3751690 & -0.3073280 & -0.4841390 \\
$\mathrm{C}$ & 2.9809700 & 1.7926810 & 0.7123870 \\
$\mathrm{C}$ & 3.7318490 & 0.6165470 & 0.7465390 \\
$\mathrm{C}$ & 2.9168610 & -0.3974830 & 0.2654680 \\
$\mathrm{C}$ & 0.5054100 & -0.3720150 & -0.5154400 \\
$\mathrm{~F}$ & 0.8444400 & 3.0000300 & -1.4223540 \\
$\mathrm{~B}$ & -1.9088570 & -0.4641450 & -1.2997630 \\
$\mathrm{~F}$ & -1.7801810 & -1.8166030 & -1.0918960 \\
$\mathrm{~F}$ & -1.9371940 & -0.1324490 & -2.6303260 \\
$\mathrm{C}$ & -4.3494380 & 1.7270850 & 0.4439830 \\
$\mathrm{C}$ & -5.1737100 & 0.6549230 & 0.1358080 \\
$\mathrm{H}$ & -1.8346640 & 3.0838820 & 0.4728170 \\
$\mathrm{H}$ & -4.6481000 & -1.2676010 & -0.8890200 \\
$\mathrm{H}$ & 3.2653420 & 2.7962840 & 0.9818230 \\
$\mathrm{H}$ & 4.7490960 & 0.5287230 & 1.0857500 \\
\hline & & &
\end{tabular}

\begin{tabular}{rrrr}
\hline$H$ & 3.1578870 & -1.4413000 & 0.1540520 \\
$H$ & 0.4775880 & -1.4092710 & -0.8172530 \\
$H$ & -4.6202180 & 2.6467530 & 0.9348800 \\
$H$ & -6.2281050 & 0.5651170 & 0.3298490 \\
$\mathrm{~F}$ & 0.4223800 & 3.4830620 & 0.7723120 \\
\hline
\end{tabular}

1, So, UMP2/6-31G, E(scf) = -1050.1213125

\begin{tabular}{rrrr}
\hline & $\mathbf{x}(\AA)$ & $\mathbf{y}(\AA)$ & $\mathbf{z}(\AA)$ \\
\hline N & -3.1202450 & 0.1344340 & -0.5679040 \\
$\mathrm{C}$ & -3.0836010 & 1.4298940 & -0.0179570 \\
$\mathrm{~N}$ & -0.6336260 & 0.2668230 & -0.7254150 \\
$\mathrm{~N}$ & -0.6758650 & 1.6222440 & -0.2863760 \\
$\mathrm{C}$ & -1.8649630 & 2.1305180 & 0.0615070 \\
$\mathrm{C}$ & 1.7111540 & 0.1660210 & -0.0944970 \\
$\mathrm{~N}$ & 1.7634950 & 1.5348000 & 0.2305510 \\
$\mathrm{~B}$ & 0.6744310 & 2.5216610 & -0.2442480 \\
$\mathrm{C}$ & -4.3940760 & -0.3652770 & -0.4228640 \\
$\mathrm{C}$ & 2.9872100 & 1.8014510 & 0.8004390 \\
$\mathrm{C}$ & 3.7318920 & 0.5938480 & 0.8710140 \\
$\mathrm{C}$ & 2.9413680 & -0.4355650 & 0.3159140 \\
$\mathrm{C}$ & 0.5220480 & -0.3971620 & -0.5956190 \\
$\mathrm{~F}$ & 0.9340380 & 3.0308740 & -1.5565230 \\
$\mathrm{~B}$ & -1.9404300 & -0.4295560 & -1.3896780 \\
$\mathrm{~F}$ & -1.7905100 & -1.8447740 & -1.2254600 \\
$\mathrm{~F}$ & -2.0115370 & -0.0602210 & -2.7707150 \\
$\mathrm{C}$ & -4.3794790 & 1.7262450 & 0.5079740 \\
$\mathrm{C}$ & -5.1907140 & 0.6005440 & 0.2482140 \\
$\mathrm{H}$ & -1.8218190 & 3.1543050 & 0.4273360 \\
$\mathrm{H}$ & -4.6568350 & -1.3441790 & -0.8079540 \\
$\mathrm{H}$ & 3.2559600 & 2.8082300 & 1.1000270 \\
$\mathrm{H}$ & 4.7282410 & 0.4923910 & 1.2872560 \\
$\mathrm{H}$ & 3.2005950 & -1.4855850 & 0.2160670 \\
$\mathrm{H}$ & 0.4752920 & -1.4379590 & -0.9092910 \\
$\mathrm{H}$ & -4.6669720 & 2.6445550 & 1.0117270 \\
$\mathrm{H}$ & -6.2349460 & 0.4793880 & 0.5149780 \\
$\mathrm{~F}$ & 0.4470150 & 3.5756890 & 0.6988990 \\
\hline & & &
\end{tabular}

1, So, UMP2/6-31G(d), E(scf) $=-1051.3523978$

\begin{tabular}{rrrr}
\hline & $\mathbf{x}(\AA)$ & $\mathbf{y}(\AA)$ & \multicolumn{1}{c}{$\mathbf{z}(\AA)$} \\
\hline N & -3.08958565 & 0.13224313 & -0.5546996 \\
$\mathrm{C}$ & -3.05161123 & 1.39553655 & 0.0007241 \\
$\mathrm{~N}$ & -0.63626021 & 0.28816811 & -0.7327478 \\
$\mathrm{~N}$ & -0.67155793 & 1.60913690 & -0.3048287 \\
$\mathrm{C}$ & -1.84671810 & 2.10343558 & 0.0496940 \\
$\mathrm{C}$ & 1.67558595 & 0.18439873 & -0.0632849 \\
$\mathrm{~N}$ & 1.73124932 & 1.53042445 & 0.2382979 \\
$\mathrm{~B}$ & 0.66816341 & 2.50846756 & -0.2965106 \\
$\mathrm{C}$ & -4.34297719 & -0.35707339 & -0.4061746 \\
$\mathrm{C}$ & 2.93486939 & 1.78765834 & 0.8018548 \\
$\mathrm{C}$ & 3.66844993 & 0.59369246 & 0.8927057 \\
$\mathrm{C}$ & 2.88644444 & -0.42108921 & 0.3449060 \\
$\mathrm{C}$ & 0.50425216 & -0.36768776 & -0.5909536 \\
$\mathrm{~F}$ & 0.93549870 & 2.89131555 & -1.5921380 \\
$\mathrm{~B}$ & -1.92837420 & -0.38932962 & -1.4220072 \\
$\mathrm{~F}$ & -1.78108823 & -1.75888244 & -1.3213107 \\
$\mathrm{~F}$ & -2.01429654 & 0.07114094 & -2.7170626 \\
$\mathrm{C}$ & -4.32804484 & 1.69549360 & 0.5300164 \\
$\mathrm{C}$ & -5.13047198 & 0.58541316 & 0.2746426 \\
$\mathrm{H}$ & -1.80560061 & 3.12628039 & 0.4116826 \\
$\mathrm{H}$ & -4.60367756 & -1.33214871 & -0.7979679 \\
$\mathrm{H}$ & 3.20258183 & 2.79543800 & 1.0933260 \\
$\mathrm{H}$ & 4.65852847 & 0.49408106 & 1.3183070 \\
$\mathrm{H}$ & 3.12880887 & -1.47370537 & 0.2605330 \\
$\mathrm{H}$ & 0.46004507 & -1.40574151 & -0.9062862 \\
$\mathrm{H}$ & -4.60087473 & 2.60631179 & 1.0494719 \\
$\mathrm{H}$ & -6.16946016 & 0.45695065 & 0.5490829 \\
\hline & & &
\end{tabular}




\begin{tabular}{llll} 
F & 0.45323917 & 3.56371076 & 0.5681300 \\
\hline
\end{tabular}

1, So, MP2/6-31G(d,p), E(scf) = -1051.4147470

\begin{tabular}{rrrr}
\hline & $\mathbf{x}(\AA)$ & $\mathbf{y}(\AA)$ & $\mathbf{z}(\AA)$ \\
\hline $\mathrm{N}$ & -3.0892720 & 0.1315380 & -0.5547040 \\
$\mathrm{C}$ & -3.0517890 & 1.3954390 & -0.0014690 \\
$\mathrm{~N}$ & -0.6362480 & 0.2889330 & -0.7334000 \\
$\mathrm{~N}$ & -0.6717020 & 1.6090780 & -0.3056410 \\
$\mathrm{C}$ & -1.8467690 & 2.1033340 & 0.0478710 \\
$\mathrm{C}$ & 1.6756610 & 0.1857170 & -0.0645390 \\
$\mathrm{~N}$ & 1.7306260 & 1.5310230 & 0.2389970 \\
$\mathrm{~B}$ & 0.6673650 & 2.5105070 & -0.2941730 \\
$\mathrm{C}$ & -4.3423450 & -0.3577190 & -0.4045620 \\
$\mathrm{C}$ & 2.9342600 & 1.7874720 & 0.8025380 \\
$\mathrm{C}$ & 3.6680850 & 0.5938190 & 0.8916870 \\
$\mathrm{C}$ & 2.8861480 & -0.4202020 & 0.3430540 \\
$\mathrm{C}$ & 0.5042890 & -0.3663340 & -0.5924520 \\
$\mathrm{~F}$ & 0.9354840 & 2.8957440 & -1.5885480 \\
$\mathrm{~B}$ & -1.9279820 & -0.3921920 & -1.4214090 \\
$\mathrm{~F}$ & -1.7777530 & -1.7605320 & -1.3161680 \\
$\mathrm{~F}$ & -2.0146170 & 0.0654470 & -2.7170510 \\
$\mathrm{C}$ & -4.3273640 & 1.6954050 & 0.5291150 \\
$\mathrm{C}$ & -5.1296550 & 0.5851740 & 0.2754030 \\
$\mathrm{H}$ & -1.8043070 & 3.1217290 & 0.4091500 \\
$\mathrm{H}$ & -4.6023400 & -1.3289610 & -0.7932750 \\
$\mathrm{H}$ & 3.2010530 & 2.7903830 & 1.0940910 \\
$\mathrm{H}$ & 4.6539030 & 0.4945830 & 1.3149780 \\
$\mathrm{H}$ & 3.1258930 & -1.4681970 & 0.2570820 \\
$\mathrm{H}$ & 0.4586480 & -1.4002320 & -0.9061630 \\
$\mathrm{H}$ & -4.5971610 & 2.6033490 & 1.0448670 \\
$\mathrm{H}$ & -6.1638070 & 0.4570600 & 0.5492280 \\
$\mathrm{~F}$ & 0.4488140 & 3.5622730 & 0.5728980 \\
\hline & & & \\
\hline
\end{tabular}

1, So, MP2/cc-pVTZ, E(scf) = -1052.5194405

\begin{tabular}{rrrr}
\hline & $\mathbf{x}(\AA)$ & \multicolumn{1}{c}{$\mathbf{y}(\AA \mathbf{)})$} & \multicolumn{1}{c}{$\mathbf{z ( \AA )}$} \\
\hline $\mathrm{N}$ & -3.1030120 & 0.1182330 & -0.5406430 \\
$\mathrm{C}$ & -3.0592230 & 1.3769590 & 0.0089940 \\
$\mathrm{~N}$ & -0.6361200 & 0.2747970 & -0.6758400 \\
$\mathrm{~N}$ & -0.6795040 & 1.5866830 & -0.2514400 \\
$\mathrm{C}$ & -1.8566490 & 2.0808650 & 0.0732810 \\
$\mathrm{C}$ & 1.6814380 & 0.1942970 & -0.0455110 \\
$\mathrm{~N}$ & 1.7423740 & 1.5330660 & 0.2584470 \\
$\mathrm{~B}$ & 0.6496500 & 2.5083740 & -0.2186070 \\
$\mathrm{C}$ & -4.3701240 & -0.3388510 & -0.4395710 \\
$\mathrm{C}$ & 2.9669430 & 1.7916520 & 0.7666570 \\
$\mathrm{C}$ & 3.7115840 & 0.6037720 & 0.8136190 \\
$\mathrm{C}$ & 2.9116720 & -0.4090710 & 0.2930800 \\
$\mathrm{C}$ & 0.5101390 & -0.3635840 & -0.5579850 \\
$\mathrm{~F}$ & 0.8887480 & 2.9425630 & -1.4940550 \\
$\mathrm{~B}$ & -1.9200230 & -0.4356180 & -1.3571220 \\
$\mathrm{~F}$ & -1.7732920 & -1.7893570 & -1.2092420 \\
$\mathrm{~F}$ & -1.9790380 & -0.0289680 & -2.6623460 \\
$\mathrm{C}$ & -4.3459000 & 1.7167940 & 0.4792550 \\
$\mathrm{C}$ & -5.1627480 & 0.6249570 & 0.2011900 \\
$\mathrm{H}$ & -1.8266480 & 3.1001750 & 0.4310930 \\
$\mathrm{H}$ & -4.6363140 & -1.3057840 & -0.8328460 \\
$\mathrm{H}$ & 3.2406140 & 2.7935700 & 1.0525700 \\
$\mathrm{H}$ & 4.7159170 & 0.5101740 & 1.1894510 \\
$\mathrm{H}$ & 3.1489090 & -1.4544990 & 0.1856820 \\
$\mathrm{H}$ & 0.4771740 & -1.3967410 & -0.8733010 \\
$\mathrm{H}$ & -4.6103480 & 2.6351390 & 0.9766950 \\
$\mathrm{H}$ & -6.2093260 & 0.5213570 & 0.4306190 \\
$\mathrm{~F}$ & 0.4302240 & 3.5226830 & 0.6752820 \\
\hline & & &
\end{tabular}

1, $S_{0}, \operatorname{CASSCF}(16,14) / 6-31 G(d), E(s c f)=-1048.7243789$

\begin{tabular}{|c|c|c|c|}
\hline & $x(\AA)$ & $y(\AA)$ & $z(\AA)$ \\
\hline B & 0.6723977 & 2.5135659 & -0.2225140 \\
\hline C & 2.9733454 & 1.7951378 & 0.7427529 \\
\hline $\mathrm{H}$ & 3.2560217 & 2.7909615 & 1.0121646 \\
\hline C & 3.7086296 & 0.6131771 & 0.8148527 \\
\hline $\mathrm{H}$ & 4.7071106 & 0.5161261 & 1.1876796 \\
\hline C & 2.8894615 & -0.4048955 & 0.3136133 \\
\hline $\mathrm{H}$ & 3.1203738 & -1.4473603 & 0.2274710 \\
\hline C & 1.6782253 & 0.2015394 & -0.0357405 \\
\hline C & -1.8385697 & 2.0692694 & 0.0823475 \\
\hline $\mathrm{H}$ & -1.8185575 & 3.0752875 & 0.4490060 \\
\hline $\mathrm{F}$ & 0.8812610 & 2.9658827 & -1.4842398 \\
\hline$N$ & 1.7531769 & 1.5385976 & 0.2402195 \\
\hline $\mathrm{N}$ & -0.6802224 & 1.5923536 & -0.2607706 \\
\hline B & -1.9416703 & -0.4367115 & -1.3662804 \\
\hline C & -4.3733765 & -0.3270044 & -0.4624658 \\
\hline $\mathrm{H}$ & -4.6466771 & -1.2789580 & -0.8665011 \\
\hline C & -5.1597158 & 0.6170520 & 0.1957868 \\
\hline $\mathrm{H}$ & -6.2003417 & 0.5179300 & 0.4252545 \\
\hline C & -4.3263570 & 1.7009135 & 0.4949611 \\
\hline $\mathrm{H}$ & -4.5871184 & 2.6042206 & 1.0082964 \\
\hline C & -3.0569268 & 1.3654657 & 0.0123380 \\
\hline C & 0.4906495 & -0.3589098 & -0.5458420 \\
\hline $\mathrm{H}$ & 0.4659085 & -1.3869493 & -0.8449004 \\
\hline $\mathrm{F}$ & -1.9712882 & -0.0543984 & -2.6674725 \\
\hline $\mathrm{N}$ & -3.1111685 & 0.1251050 & -0.5604241 \\
\hline $\mathrm{N}$ & -0.6338848 & 0.2755926 & -0.6864856 \\
\hline$F$ & -1.7847783 & -1.7770330 & -1.2012944 \\
\hline $\mathrm{F}$ & 0.4412071 & 3.5076854 & 0.6755880 \\
\hline
\end{tabular}

1, $T_{1}, \operatorname{CASSCF}(16,14) / 6-31 G(d), E(s c f)=-1048.63326310$

\begin{tabular}{rrrr}
\hline & $\mathbf{x}(\AA)$ & $\mathbf{y}(\AA)$ & $\mathbf{z}(\AA)$ \\
\hline B & 0.6326922 & 2.4700704 & 0.0560421 \\
$\mathrm{C}$ & 3.0262620 & 1.7711195 & 0.7444983 \\
$\mathrm{H}$ & 3.2732093 & 2.7407658 & 1.1198785 \\
$\mathrm{C}$ & 3.8270330 & 0.6311686 & 0.6067240 \\
$\mathrm{H}$ & 4.8568827 & 0.5554350 & 0.8879228 \\
$\mathrm{C}$ & 3.0339051 & -0.3658695 & 0.0541552 \\
$\mathrm{H}$ & 3.3068973 & -1.3744111 & -0.1780469 \\
$\mathrm{C}$ & 1.7281544 & 0.2043999 & -0.1303681 \\
$\mathrm{C}$ & -1.9048645 & 2.1082992 & 0.0510179 \\
$\mathrm{H}$ & -1.8547394 & 3.1224708 & 0.3765850 \\
$\mathrm{~F}$ & 0.7520135 & 3.1745245 & -1.1004178 \\
$\mathrm{~N}$ & 1.7638205 & 1.4945766 & 0.2976807 \\
$\mathrm{~N}$ & -0.6961240 & 1.5109577 & -0.1501822 \\
$\mathrm{~B}$ & -1.9391489 & -0.5664102 & -1.1129490 \\
$\mathrm{C}$ & -4.4269853 & -0.3065139 & -0.4535487 \\
$\mathrm{H}$ & -4.6792512 & -1.3009431 & -0.7528475 \\
$\mathrm{C}$ & -5.2501112 & 0.7293290 & 0.0038302 \\
$\mathrm{H}$ & -6.3094025 & 0.6679408 & 0.1435886 \\
$\mathrm{C}$ & -4.4352888 & 1.8274202 & 0.2461742 \\
$\mathrm{H}$ & -4.7180058 & 2.7906803 & 0.6173883 \\
$\mathrm{C}$ & -3.0944145 & 1.4207277 & -0.0714732 \\
$\mathrm{C}$ & 0.5618541 & -0.3748808 & -0.5853035 \\
$\mathrm{H}$ & 0.5127719 & -1.3841912 & -0.9257944 \\
$\mathrm{~F}$ & -1.8829174 & -0.4532500 & -2.4664357 \\
$\mathrm{~N}$ & -3.1307450 & 0.1274421 & -0.4899788 \\
$\mathrm{~N}$ & -0.6354034 & 0.2759228 & -0.5482292 \\
$\mathrm{~F}$ & -1.7988253 & -1.8504030 & -0.6912531 \\
$\mathrm{~F}$ & 0.3878471 & 3.2672653 & 1.1287430 \\
\hline & & &
\end{tabular}

$\mathbf{1}, \mathbf{S}_{1}, \operatorname{CASSCF}(16,14) / 6-31 G(d), E(s c f)=-1048.59326127$

\begin{tabular}{cccc}
\hline & $\mathbf{x}(\AA)$ & $\mathbf{y}(\AA)$ & $\mathbf{z}(\AA)$ \\
\hline B & 0.5973158 & 2.4480464 & 0.0338275 \\
C & 3.0001010 & 1.7806438 & 0.7069055 \\
H & 3.2437477 & 2.7626570 & 1.0533000 \\
\hline
\end{tabular}




\begin{tabular}{rrrr}
\hline $\mathrm{C}$ & 3.8274053 & 0.6335100 & 0.6061186 \\
$\mathrm{H}$ & 4.8569028 & 0.5841179 & 0.8933757 \\
$\mathrm{C}$ & 3.0443155 & -0.3861804 & 0.0771765 \\
$\mathrm{H}$ & 3.3295364 & -1.3973796 & -0.1253616 \\
$\mathrm{C}$ & 1.7514052 & 0.1658610 & -0.1251063 \\
$\mathrm{C}$ & -1.9024878 & 2.1204257 & 0.0802678 \\
$\mathrm{H}$ & -1.8444501 & 3.1321153 & 0.4130376 \\
$\mathrm{~F}$ & 0.7547495 & 3.1500153 & -1.1230512 \\
$\mathrm{~N}$ & 1.7753186 & 1.4888758 & 0.2728853 \\
$\mathrm{~N}$ & -0.6963009 & 1.5105274 & -0.1265946 \\
$\mathrm{~B}$ & -1.9021911 & -0.5370197 & -1.1133513 \\
$\mathrm{C}$ & -4.3959319 & -0.2932461 & -0.4859246 \\
$\mathrm{H}$ & -4.6407702 & -1.2808171 & -0.8152581 \\
$\mathrm{C}$ & -5.2503085 & 0.7278056 & 0.0019238 \\
$\mathrm{H}$ & -6.3089333 & 0.6415407 & 0.1311263 \\
$\mathrm{C}$ & -4.4493388 & 1.8307786 & 0.2751970 \\
$\mathrm{H}$ & -4.7480208 & 2.7792947 & 0.6701691 \\
$\mathrm{C}$ & -3.1197038 & 1.4497733 & -0.0475885 \\
$\mathrm{C}$ & 0.5563130 & -0.4017429 & -0.5693144 \\
$\mathrm{H}$ & 0.4983793 & -1.4129435 & -0.9035852 \\
$\mathrm{~F}$ & -1.8837826 & -0.4200493 & -2.4705147 \\
$\mathrm{~N}$ & -3.1392547 & 0.1470661 & -0.5079256 \\
$\mathrm{~N}$ & -0.6382143 & 0.2624340 & -0.5290811 \\
$\mathrm{~F}$ & -1.8139138 & -1.8346824 & -0.7053706 \\
$\mathrm{~F}$ & 0.4052282 & 3.2622157 & 1.1101179 \\
\hline & & & \\
\hline
\end{tabular}

2, So, UB3LYP/6-31G(d), E(scf) $=-660.4867945$

\begin{tabular}{rrrr}
\hline & \multicolumn{1}{c}{$\mathbf{x ( \AA )}$} & $\mathbf{y}(\AA \AA)$ & \multicolumn{1}{c}{$\mathbf{z ( \AA ̊ )}$} \\
\hline $\mathrm{N}$ & -3.1615260 & 0.1035130 & -0.5189750 \\
$\mathrm{C}$ & -3.1851210 & 1.4220720 & -0.0998590 \\
$\mathrm{~N}$ & -0.6754820 & 0.3520490 & -0.4860190 \\
$\mathrm{~N}$ & -0.7532960 & 1.7135750 & -0.1816310 \\
$\mathrm{C}$ & -1.9740470 & 2.1541690 & -0.0191900 \\
$\mathrm{C}$ & -4.4266480 & -0.3768080 & -0.4672800 \\
$\mathrm{C}$ & 0.4666320 & -0.2289010 & -0.3242530 \\
$\mathrm{~B}$ & -1.9077590 & -0.5009160 & -1.1516040 \\
$\mathrm{~F}$ & -1.7310100 & -1.8255290 & -0.8114920 \\
$\mathrm{~F}$ & -1.8421820 & -0.2872210 & -2.5100210 \\
$\mathrm{C}$ & -4.5055480 & 1.7633420 & 0.2273050 \\
$\mathrm{C}$ & -5.2873330 & 0.6203510 & -0.0011590 \\
$\mathrm{H}$ & -2.0304020 & 3.2115490 & 0.2326370 \\
$\mathrm{H}$ & -4.6423280 & -1.3925160 & -0.7693380 \\
$\mathrm{H}$ & 0.4688030 & -1.2860340 & -0.5731060 \\
$\mathrm{H}$ & -4.8369080 & 2.7223920 & 0.6038770 \\
$\mathrm{H}$ & -6.3520830 & 0.5152650 & 0.1561750 \\
$\mathrm{C}$ & 1.7079480 & 0.4219080 & 0.1715930 \\
$\mathrm{H}$ & 1.8876070 & 0.1218470 & 1.2137120 \\
$\mathrm{H}$ & 2.5666060 & 0.0713350 & -0.4114070 \\
$\mathrm{H}$ & 1.6303810 & 1.5082360 & 0.1302380 \\
\hline & & &
\end{tabular}

2, So, UB3LYP/cc-pVTZ, E(scf) $=-660.7330200$

\begin{tabular}{crrr}
\hline & $\mathbf{x}(\AA)$ & $\mathbf{y}(\AA)$ & \multicolumn{1}{c}{$\mathbf{z}(\mathbf{\AA})$} \\
\hline $\mathrm{N}$ & -3.1721630 & 0.1019480 & -0.5079510 \\
$\mathrm{C}$ & -3.1897650 & 1.4146970 & -0.0874050 \\
$\mathrm{~N}$ & -0.6740250 & 0.3456580 & -0.4484640 \\
$\mathrm{~N}$ & -0.7673570 & 1.6976750 & -0.1254400 \\
$\mathrm{C}$ & -1.9817400 & 2.1384450 & 0.0242430 \\
$\mathrm{C}$ & -4.4413810 & -0.3616120 & -0.4905050 \\
$\mathrm{C}$ & 0.4761390 & -0.2151930 & -0.3290860 \\
$\mathrm{~B}$ & -1.9084350 & -0.5267680 & -1.0923110 \\
$\mathrm{~F}$ & -1.7426540 & -1.8409870 & -0.7113520 \\
$\mathrm{~F}$ & -1.8211650 & -0.3676260 & -2.4590030 \\
$\mathrm{C}$ & -4.5085650 & 1.7707990 & 0.2048710 \\
$\mathrm{C}$ & -5.2966830 & 0.6419270 & -0.0461850 \\
$\mathrm{H}$ & -2.0415560 & 3.1882840 & 0.2879800 \\
$\mathrm{H}$ & -4.6647240 & -1.3689440 & -0.7984640 \\
\hline & & &
\end{tabular}

\begin{tabular}{rrrr}
\hline$H$ & 0.4918540 & -1.2646030 & -0.5948600 \\
$H$ & -4.8359280 & 2.7297210 & 0.5707350 \\
$H$ & -6.3617620 & 0.5512120 & 0.0800180 \\
C & 1.7203610 & 0.4374380 & 0.1370690 \\
H & 1.9739190 & 0.0586650 & 1.1320530 \\
H & 2.5450010 & 0.1571950 & -0.5205400 \\
H & 1.6169340 & 1.5157480 & 0.1848010 \\
\hline
\end{tabular}

2, So, UM06-2X/6-31G(d), E(scf) $=-660.2346440$

\begin{tabular}{rrrr}
\hline & $\mathbf{x}(\mathbf{\AA})$ & \multicolumn{1}{c}{$\mathbf{y}(\AA)$} & \multicolumn{1}{c}{$\mathbf{z}(\AA)$} \\
\hline N & -3.1495410 & 0.1020430 & -0.5406500 \\
$\mathrm{C}$ & -3.1611160 & 1.3980870 & -0.0798190 \\
$\mathrm{~N}$ & -0.6797990 & 0.3403070 & -0.5256240 \\
$\mathrm{~N}$ & -0.7367650 & 1.6904180 & -0.1912630 \\
$\mathrm{C}$ & -1.9434400 & 2.1307290 & 0.0007320 \\
$\mathrm{C}$ & -4.4069270 & -0.3808400 & -0.4745010 \\
$\mathrm{C}$ & 0.4481190 & -0.2470430 & -0.3555370 \\
$\mathrm{~B}$ & -1.9108350 & -0.4750270 & -1.2220020 \\
$\mathrm{~F}$ & -1.7266300 & -1.8067290 & -0.9460950 \\
$\mathrm{~F}$ & -1.8564690 & -0.1879260 & -2.5600390 \\
$\mathrm{C}$ & -4.4643290 & 1.7292460 & 0.2926580 \\
$\mathrm{C}$ & -5.2524270 & 0.5957950 & 0.0463310 \\
$\mathrm{H}$ & -1.9958950 & 3.1810030 & 0.2796220 \\
$\mathrm{H}$ & -4.6257500 & -1.3866070 & -0.8057490 \\
$\mathrm{H}$ & 0.4559470 & -1.2984360 & -0.6282300 \\
$\mathrm{H}$ & -4.7835270 & 2.6753400 & 0.7085750 \\
$\mathrm{H}$ & -6.3117630 & 0.4856960 & 0.2267980 \\
$\mathrm{C}$ & 1.6585560 & 0.4285670 & 0.1842390 \\
$\mathrm{H}$ & 1.5246370 & 0.6254010 & 1.2537800 \\
$\mathrm{H}$ & 2.5412440 & -0.1956800 & 0.0461030 \\
$\mathrm{H}$ & 1.7930130 & 1.3993350 & -0.2991250 \\
\hline
\end{tabular}

2, So, UM06-2X/cc-pVTZ, E(scf) $=-660.492255$

\begin{tabular}{rrrr}
\hline & $\mathbf{x}(\AA)$ & \multicolumn{1}{c}{$\mathbf{y}(\AA ̊)$} & \multicolumn{1}{c}{$\mathbf{z}(\AA)$} \\
\hline $\mathrm{N}$ & -3.1554100 & 0.0988350 & -0.5282130 \\
$\mathrm{C}$ & -3.1634800 & 1.3901060 & -0.0643190 \\
$\mathrm{~N}$ & -0.6787130 & 0.3307500 & -0.4952360 \\
$\mathrm{~N}$ & -0.7469410 & 1.6746430 & -0.1445060 \\
$\mathrm{C}$ & -1.9482820 & 2.1158340 & 0.0393480 \\
$\mathrm{C}$ & -4.4180380 & -0.3688770 & -0.4920270 \\
$\mathrm{C}$ & 0.4571220 & -0.2395700 & -0.3619680 \\
$\mathrm{~B}$ & -1.9107420 & -0.4927120 & -1.1776300 \\
$\mathrm{~F}$ & -1.7369450 & -1.8205860 & -0.8854570 \\
$\mathrm{~F}$ & -1.8429270 & -0.2365210 & -2.5218320 \\
$\mathrm{C}$ & -4.4660630 & 1.7339330 & 0.2789260 \\
$\mathrm{C}$ & -5.2603160 & 0.6124220 & 0.0121570 \\
$\mathrm{H}$ & -2.0034440 & 3.1595830 & 0.3277160 \\
$\mathrm{H}$ & -4.6418560 & -1.3668660 & -0.8307820 \\
$\mathrm{H}$ & 0.4749120 & -1.2840910 & -0.6488660 \\
$\mathrm{H}$ & -4.7814110 & 2.6798920 & 0.6870770 \\
$\mathrm{H}$ & -6.3205810 & 0.5142280 & 0.1670000 \\
$\mathrm{C}$ & 1.6699700 & 0.4391430 & 0.1537010 \\
$\mathrm{H}$ & 1.5546550 & 0.6276970 & 1.2230300 \\
$\mathrm{H}$ & 2.5500110 & -0.1759740 & -0.0061410 \\
$\mathrm{H}$ & 1.7847830 & 1.4118100 & -0.3217750 \\
\hline
\end{tabular}

2, $S_{0}, U \omega B 97 X D / 6-31 G(d), E(s c f)=-660.2793993$

\begin{tabular}{cccc}
\hline & $\mathbf{x}(\AA)$ & $\mathbf{y}(\AA)$ & $\mathbf{z}(\AA)$ \\
\hline $\mathrm{N}$ & -3.1500070 & 0.1046870 & -0.5324990 \\
$\mathrm{C}$ & -3.1683810 & 1.4110270 & -0.0981620 \\
$\mathrm{~N}$ & -0.6800610 & 0.3503440 & -0.5230650 \\
$\mathrm{~N}$ & -0.7476060 & 1.7129210 & -0.2270650 \\
\hline
\end{tabular}




\begin{tabular}{rrrr}
\hline C & -1.9559700 & 2.1501020 & -0.0402430 \\
C & -4.4077240 & -0.3791810 & -0.4615090 \\
C & 0.4482600 & -0.2325210 & -0.3332780 \\
B & -1.9103960 & -0.4844420 & -1.1945810 \\
F & -1.7273730 & -1.8152190 & -0.8848530 \\
F & -1.8588210 & -0.2399290 & -2.5469750 \\
C & -4.4731290 & 1.7453100 & 0.2610170 \\
C & -5.2569200 & 0.6043370 & 0.0345910 \\
H & -2.0121450 & 3.2078620 & 0.2061570 \\
H & -4.6244040 & -1.3914240 & -0.7720310 \\
H & 0.4537070 & -1.2907470 & -0.5757090 \\
H & -4.7984290 & 2.6981850 & 0.6567470 \\
H & -6.3172040 & 0.4950240 & 0.2125110 \\
C & 1.6707480 & 0.4241630 & 0.1931220 \\
H & 1.7262790 & 0.2648620 & 1.2775740 \\
H & 2.5591700 & -0.0306150 & -0.2518620 \\
H & 1.6467100 & 1.4989340 & 0.0103160 \\
\hline
\end{tabular}

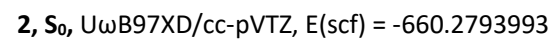

\begin{tabular}{rrrr}
\hline & \multicolumn{1}{c}{$\mathbf{x}(\AA \mathbf{)})$} & $\mathbf{y}(\AA)$ & \multicolumn{1}{c}{$\mathbf{z}(\AA)$} \\
\hline $\mathrm{N}$ & -3.1595430 & 0.1053610 & -0.5186540 \\
$\mathrm{C}$ & -3.1778940 & 1.4117560 & -0.1018530 \\
$\mathrm{~N}$ & -0.6801800 & 0.3515060 & -0.4831680 \\
$\mathrm{~N}$ & -0.7648210 & 1.7112250 & -0.1906300 \\
$\mathrm{C}$ & -1.9689750 & 2.1473890 & -0.0258050 \\
$\mathrm{C}$ & -4.4188280 & -0.3681430 & -0.4699680 \\
$\mathrm{C}$ & 0.4535010 & -0.2151960 & -0.3217310 \\
$\mathrm{~B}$ & -1.9094030 & -0.5061880 & -1.1340530 \\
$\mathrm{~F}$ & -1.7363590 & -1.8258940 & -0.7842570 \\
$\mathrm{~F}$ & -1.8358670 & -0.3128370 & -2.4937150 \\
$\mathrm{C}$ & -4.4823730 & 1.7585830 & 0.2222930 \\
$\mathrm{C}$ & -5.2675530 & 0.6234570 & -0.0067080 \\
$\mathrm{H}$ & -2.0316930 & 3.2023620 & 0.2146380 \\
$\mathrm{H}$ & -4.6365150 & -1.3790460 & -0.7704810 \\
$\mathrm{H}$ & 0.4658080 & -1.2698530 & -0.5682890 \\
$\mathrm{H}$ & -4.8073700 & 2.7150930 & 0.5966080 \\
$\mathrm{H}$ & -6.3277890 & 0.5235410 & 0.1478190 \\
$\mathrm{C}$ & 1.6870160 & 0.4308470 & 0.1728120 \\
$\mathrm{H}$ & 1.9012830 & 0.0522870 & 1.1752740 \\
$\mathrm{H}$ & 2.5241360 & 0.1375240 & -0.4607070 \\
$\mathrm{H}$ & 1.5897270 & 1.5099050 & 0.2107790 \\
\hline & & & \\
\hline
\end{tabular}

2, So, UMP2/6-31G(d), E(scf) $=-656.769225736$

\begin{tabular}{rrrr}
\hline & $\mathbf{x}(\AA)$ & $\mathbf{y}(\AA)$ & $\mathbf{z}(\AA)$ \\
\hline $\mathrm{N}$ & -3.1381330 & 0.1023380 & -0.5413840 \\
$\mathrm{C}$ & -3.1561000 & 1.4087340 & -0.1020810 \\
$\mathrm{~N}$ & -0.6815620 & 0.3381830 & -0.5516110 \\
$\mathrm{~N}$ & -0.7222320 & 1.7035730 & -0.2385760 \\
$\mathrm{C}$ & -1.9454480 & 2.1452460 & -0.0423590 \\
$\mathrm{C}$ & -4.3985530 & -0.4010720 & -0.4458210 \\
$\mathrm{C}$ & 0.4507530 & -0.2586250 & -0.3444490 \\
$\mathrm{~B}$ & -1.9148320 & -0.4536210 & -1.2657860 \\
$\mathrm{~F}$ & -1.7330100 & -1.8069440 & -1.0417970 \\
$\mathrm{~F}$ & -1.8766030 & -0.1078360 & -2.6023970 \\
$\mathrm{C}$ & -4.4649250 & 1.7260690 & 0.3027680 \\
$\mathrm{C}$ & -5.2406320 & 0.5796660 & 0.0848840 \\
$\mathrm{H}$ & -1.9897600 & 3.2025500 & 0.2166050 \\
$\mathrm{H}$ & -4.6138850 & -1.4128760 & -0.7645090 \\
$\mathrm{H}$ & 0.4584430 & -1.3125410 & -0.6043300 \\
$\mathrm{H}$ & -4.7892400 & 2.6729820 & 0.7181040 \\
$\mathrm{H}$ & -6.2959310 & 0.4596590 & 0.2935900 \\
$\mathrm{C}$ & 1.6464960 & 0.4190440 & 0.2203090 \\
$\mathrm{H}$ & 1.4862240 & 0.6439950 & 1.2801410 \\
$\mathrm{H}$ & 2.5252300 & -0.2192300 & 0.1228030 \\
\hline & & &
\end{tabular}

\begin{tabular}{llll}
\hline $\mathrm{H}$ & 1.8100030 & 1.3743830 & -0.2839000 \\
\hline
\end{tabular}

2, So, UMP2/cc-pVTZ, E(scf) = -657.002651157

\begin{tabular}{rrrr}
\hline & $\mathbf{x}(\mathbf{A})$ & $\mathbf{y}(\AA)$ & $\mathbf{z}(\AA)$ \\
\hline N & -3.1485500 & 0.0945970 & -0.5228370 \\
$\mathrm{C}$ & -3.1576610 & 1.3945340 & -0.0829550 \\
$\mathrm{~N}$ & -0.6796520 & 0.3375600 & -0.5141060 \\
$\mathrm{~N}$ & -0.7320420 & 1.6901410 & -0.1940910 \\
$\mathrm{C}$ & -1.9518340 & 2.1288340 & -0.0097150 \\
$\mathrm{C}$ & -4.4170150 & -0.3842150 & -0.4667730 \\
$\mathrm{C}$ & 0.4606760 & -0.2447420 & -0.3452420 \\
$\mathrm{~B}$ & -1.9105220 & -0.4888630 & -1.2010580 \\
$\mathrm{~F}$ & -1.7312200 & -1.8218240 & -0.9268340 \\
$\mathrm{~F}$ & -1.8595040 & -0.2039970 & -2.5425510 \\
$\mathrm{C}$ & -4.4714990 & 1.7417560 & 0.2726580 \\
$\mathrm{C}$ & -5.2596230 & 0.6107610 & 0.0338430 \\
$\mathrm{H}$ & -2.0050310 & 3.1780360 & 0.2558610 \\
$\mathrm{H}$ & -4.6391290 & -1.3874830 & -0.7891830 \\
$\mathrm{H}$ & 0.4668100 & -1.2939500 & -0.6069860 \\
$\mathrm{H}$ & -4.7840080 & 2.6925650 & 0.6718810 \\
$\mathrm{H}$ & -6.3176110 & 0.5080630 & 0.2039730 \\
$\mathrm{C}$ & 1.6671310 & 0.4343050 & 0.1803310 \\
$\mathrm{H}$ & 1.6155330 & 0.4765630 & 1.2700020 \\
$\mathrm{H}$ & 2.5594660 & -0.1177830 & -0.0979870 \\
$\mathrm{H}$ & 1.7115890 & 1.4588170 & -0.1780270 \\
\hline
\end{tabular}

2, $\mathrm{S}_{0}, \operatorname{CASSCF}(10,8) / 6-31 \mathrm{G}(\mathrm{d}), \mathrm{E}(\mathrm{scf})=-656.85354412$

\begin{tabular}{rrrr}
\hline & \multicolumn{1}{c}{$\mathbf{x ( \AA ̊ )}$} & \multicolumn{1}{c}{$\mathbf{y}(\AA ̊)$} & \multicolumn{1}{c}{$\mathbf{z}(\AA ̊ \AA)$} \\
\hline N & 1.0983680 & -0.3202909 & -0.1311569 \\
$\mathrm{C}$ & 1.2699679 & 1.0221272 & 0.0851290 \\
$\mathrm{~N}$ & -1.3311660 & 0.1774667 & 0.0029713 \\
$\mathrm{~N}$ & -1.1054780 & 1.5031798 & 0.3702497 \\
$\mathrm{C}$ & 0.1341124 & 1.8482074 & 0.3907370 \\
$\mathrm{C}$ & 2.3067835 & -0.8454748 & -0.4407489 \\
$\mathrm{C}$ & -2.5064313 & -0.1015070 & -0.4185540 \\
$\mathrm{~B}$ & -0.1998931 & -1.0159669 & 0.1990238 \\
$\mathrm{~F}$ & -0.5212321 & -2.0111686 & -0.6736897 \\
$\mathrm{~F}$ & -0.3149402 & -1.4055207 & 1.4989916 \\
$\mathrm{C}$ & 2.5985655 & 1.3448700 & -0.1078845 \\
$\mathrm{C}$ & 3.2552412 & 0.1357721 & -0.4478191 \\
$\mathrm{H}$ & 0.3062711 & 2.8764044 & 0.6529464 \\
$\mathrm{H}$ & 2.4101925 & -1.8930376 & -0.6357745 \\
$\mathrm{H}$ & -2.6537225 & -1.1262465 & -0.6977971 \\
$\mathrm{H}$ & 3.0332669 & 2.3215142 & -0.0316262 \\
$\mathrm{H}$ & 4.2945097 & 0.0081040 & -0.6723757 \\
$\mathrm{C}$ & -3.6265246 & 0.8816955 & -0.5626698 \\
$\mathrm{H}$ & -3.6773289 & 1.5406148 & 0.2924422 \\
$\mathrm{H}$ & -3.4694829 & 1.4949670 & -1.4442610 \\
$\mathrm{H}$ & -4.5638806 & 0.3533352 & -0.6768577 \\
\hline
\end{tabular}

2, $\mathbf{S}_{1}, \operatorname{CASSCF}(10,8) / 6-31 G(d), E(s c f)=-656.7146714$

\begin{tabular}{rrrr}
\hline & \multicolumn{1}{c}{$\mathbf{x}(\AA) \mathbf{(})$} & $\mathbf{y}(\AA)$ & \multicolumn{1}{c}{$\mathbf{z ( \AA )})$} \\
\hline $\mathrm{N}$ & 1.1058371 & -0.3454864 & 0.0160700 \\
$\mathrm{C}$ & 1.2450693 & 0.9983863 & 0.1900821 \\
$\mathrm{~N}$ & -1.2716305 & 0.1127778 & 0.0668531 \\
$\mathrm{~N}$ & -1.0869355 & 1.3436365 & 0.6684561 \\
$\mathrm{C}$ & 0.1208874 & 1.7710740 & 0.7240787 \\
$\mathrm{C}$ & 2.2200275 & -0.8204643 & -0.5103460 \\
$\mathrm{C}$ & -2.6004061 & -0.1596887 & -0.2465756 \\
$\mathrm{~B}$ & -0.3107400 & -1.0248571 & 0.3616042 \\
$\mathrm{~F}$ & -0.4823118 & -2.0830879 & -0.4822675 \\
$\mathrm{~F}$ & -0.2500998 & -1.4081994 & 1.6747940 \\
$\mathrm{C}$ & 2.5561335 & 1.3869672 & -0.2320715 \\
$\mathrm{C}$ & 3.1636021 & 0.2623711 & -0.6757385 \\
\hline
\end{tabular}




\begin{tabular}{rrrr}
\hline $\mathrm{H}$ & 0.3007142 & 2.7281574 & 1.1750725 \\
$\mathrm{H}$ & 2.3283915 & -1.8561579 & -0.7524909 \\
$\mathrm{H}$ & -2.7468487 & -1.1630393 & -0.5955195 \\
$\mathrm{H}$ & 2.9485144 & 2.3825359 & -0.2039435 \\
$\mathrm{H}$ & 4.1532175 & 0.1617133 & -1.0715105 \\
$\mathrm{C}$ & -3.4893677 & 0.9182815 & -0.7809097 \\
$\mathrm{H}$ & -3.5210443 & 1.7742808 & -0.1175802 \\
$\mathrm{H}$ & -3.1481320 & 1.2715029 & -1.7558906 \\
$\mathrm{H}$ & -4.4976797 & 0.5383416 & -0.9008909 \\
\hline
\end{tabular}

